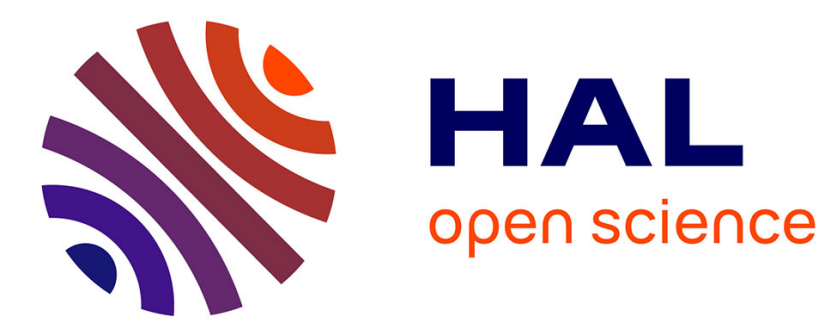

\title{
Un potier ou décorateur du Sud de la Gaule. C. CINO SENOVIR...
}

\author{
Colette Bémont
}

\section{To cite this version:}

Colette Bémont. Un potier ou décorateur du Sud de la Gaule. C. CINO SENOVIR.... Gallia - Fouilles et monuments archéologiques en France métropolitaine, 1969, 27 (2), pp.186-205. 10.3406/galia.1969.2528 . hal-01934096

\section{HAL Id: hal-01934096 https://hal.science/hal-01934096}

Submitted on 3 Mar 2020

HAL is a multi-disciplinary open access archive for the deposit and dissemination of scientific research documents, whether they are published or not. The documents may come from teaching and research institutions in France or abroad, or from public or private research centers.
L'archive ouverte pluridisciplinaire HAL, est destinée au dépôt et à la diffusion de documents scientifiques de niveau recherche, publiés ou non, émanant des établissements d'enseignement et de recherche français ou étrangers, des laboratoires publics ou privés.

\section{(ㅇ)(1) $\$$}

Distributed under a Creative Commons Attribution - NonCommercial - NoDerivatives| 4.0 


\section{Un potier ou décorateur du Sud de la Gaule : C. CINO SENOVIR...}

Grâce à deux fragments de signatures manuscrites infradécoratives, sur des bols D. 371, l'occasion m'a été donnée récemment de signaler l'existence d'un atelier apparemment peu connu : celui de C. CINO SENOV [-- ]. L'étude des tessons a permis une localisation probable : la Graufesenque ou ses environs, une datation vraisemblable : la fin $d{ }^{\text {er }}$ ou le début du ${ }_{I^{e}}{ }^{\mathrm{s}}$. et fourni quelques éléments du répertoire de ce potier ou décorateur de moules ${ }^{2}$. Lne double incertitude subsistait cependant à propos du dernier élément de l'inscription : quel était le nom complet? quelle forme grammaticale avait-il prise dans cette marque? Si l'on n'avait pas affaire à une abréviation. le nominatif pouvait indiquer l'association - plutòt, sans doute, que l'attribution d'un second cognomen gaulois à un même artisan . le grénitif, la filiation ou la dépendance ${ }^{3}$.

L'inventaire, que je viens de faire, des vases décorés de forme 37, au Musée de Saint-Rémyde-Provence $e^{4}$ et l'examen. auquel s'est livré

1 L'un, signi C. CINO SENOV . . , provient des environs de Glanum et se trouve au Musée de Saint-Rémy-de-Provence; l'autre, découvert dans la grotte de Vidauque, est au Musée de Cavaillon. II porte la marque $[$ c] CINO is - - i.

2 Il n'est pas possible, pour le moment, de savoir si celui qui a signé le moule appartient ou non à l'atelier où a été fabriqué le vase. J'ai déjà abordé cette question dans une note (Signalure inédile sur un moule de sigillee: C. CINO SENOV..., dans Gallia, XXVI, 1968, p. 303-313).

3 Ibid., p. 301-308.

4 Je remercie, a celle occasion, .H. H. Rolland,
II. A. Dumoulin, des réserves de Cavaillon ont révélé sûrement deux, très probablement, trois tessons, qui s'ajoutent à cette courte série (fig. 1). Les marques sont, malheureusement, imparfaites, mais l'une d'elles, du moins, apporte une réponse à l'une des questions en suspens. Le décor des vases enrichit le catalogue des poinçons utilisés dans cet atelier et permet une connaissance partielle de la technique de l'ornementation. Enfin, l'origine vraisemblable des motifs, comme la provenance de l'argile des vases, parait pouvoir confirmer les hypothèses proposées relativement à l'époque et au lieu de fabrication.

La première signature (fig. $2,2,3,4,5)^{5}$ est la plus nette mais la moins assurée. Elle est, en effet, réduite par une cassure à $C . C I N O$ et à l'extrémité effilée, sous l'alignement, d'un trait approximativement parallèle à celui-ci. La disposition générale de l'inscription ménage des intervalles étroits entre les différents éléments. Ilais on peut faire la même observation à propos du premier tesson de Saint-Rémy (fig. 2, 5), où l'initiale du prénom est très proche du début du cognomen. Caractéristique plus notable : le tracé élégant, la hauteur des lettres de cette signature. Je n'oserais aborder des problèmes de graphologie antique pour discuter de l'attribution de ce texte. On peut néanmoins constater la différence avec les caractères plus menus de la seule inscription de

\footnotetext{
Directeur des fouilles de Glanum, de m'avoir si aimablement donné accès à ce matériel.

5) Saint-Rémy, no 3064 , ancien fonds
} 


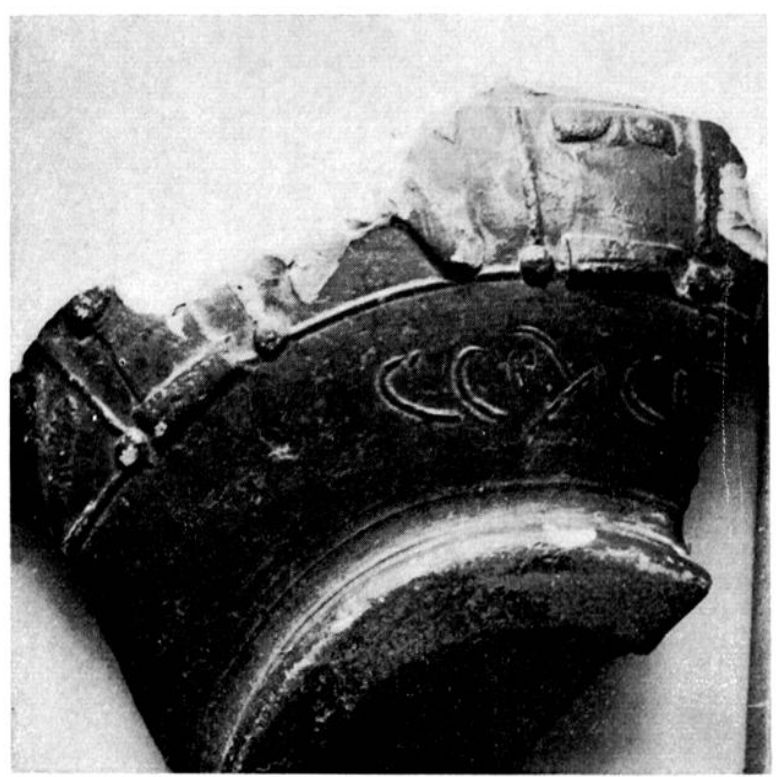

1

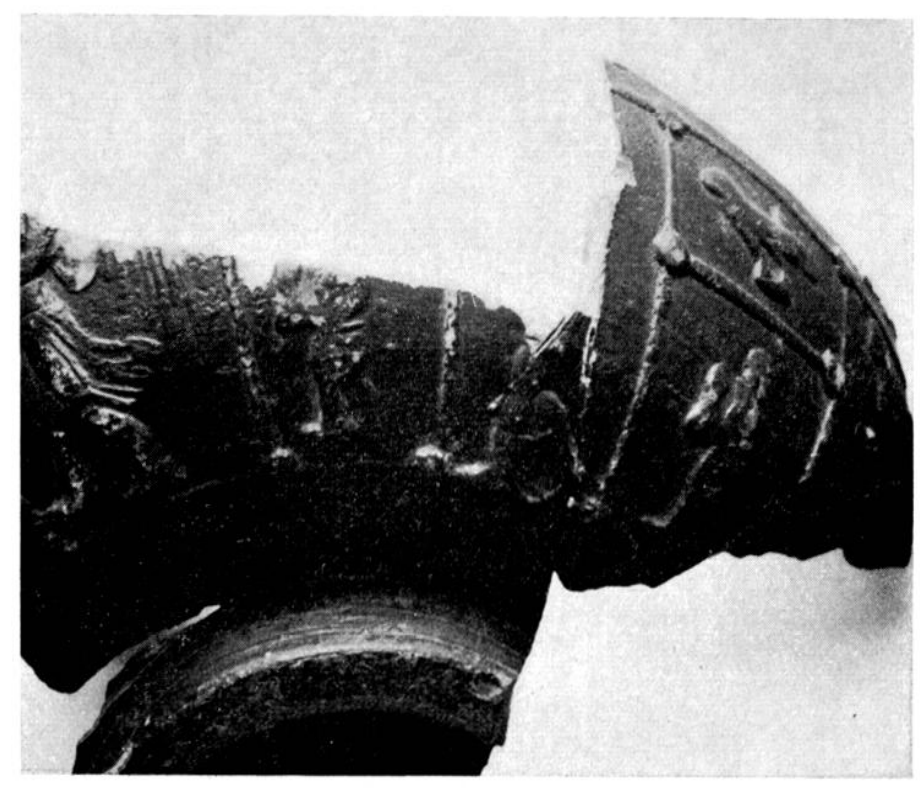

2

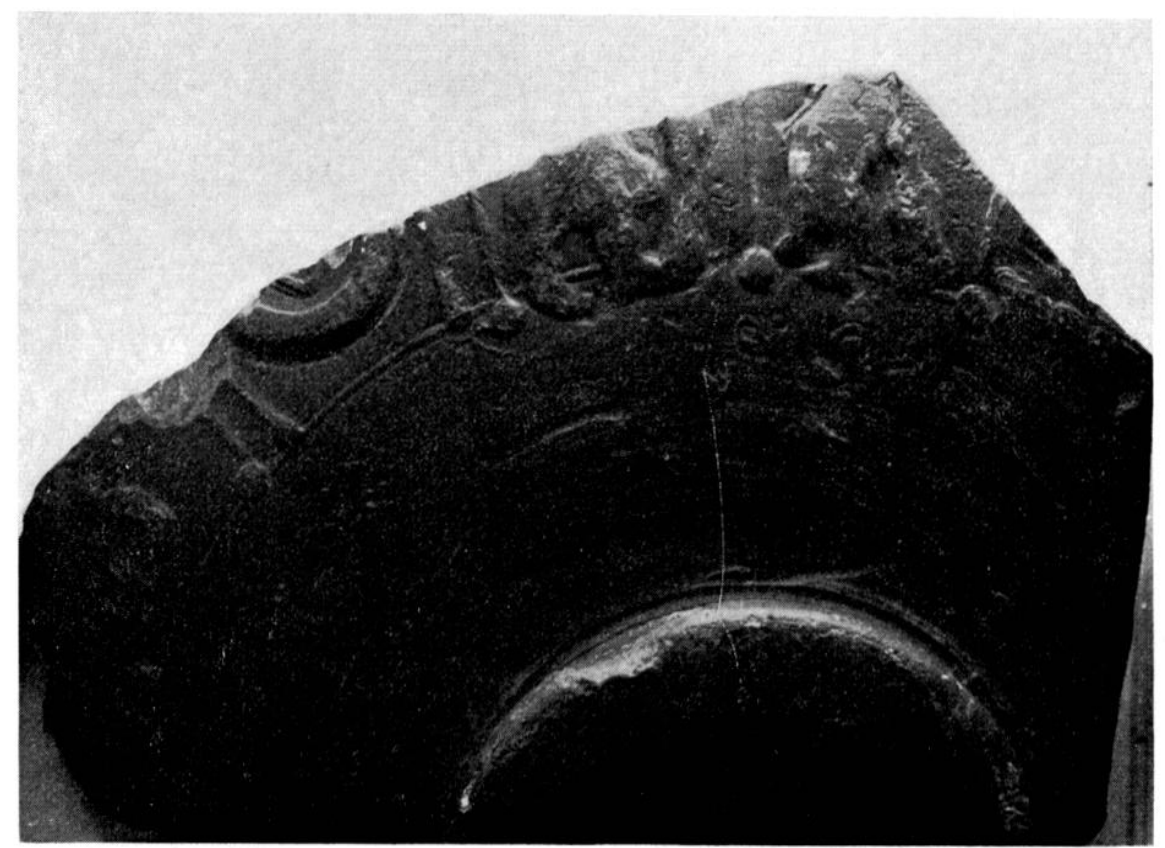

3

1 1, Saint-Rémy, no 3064 (bol C.); 2, Saint-Rémy, no 291 (bol D); 3, Cavaillon, réf. 55-12-4 (bol E) 

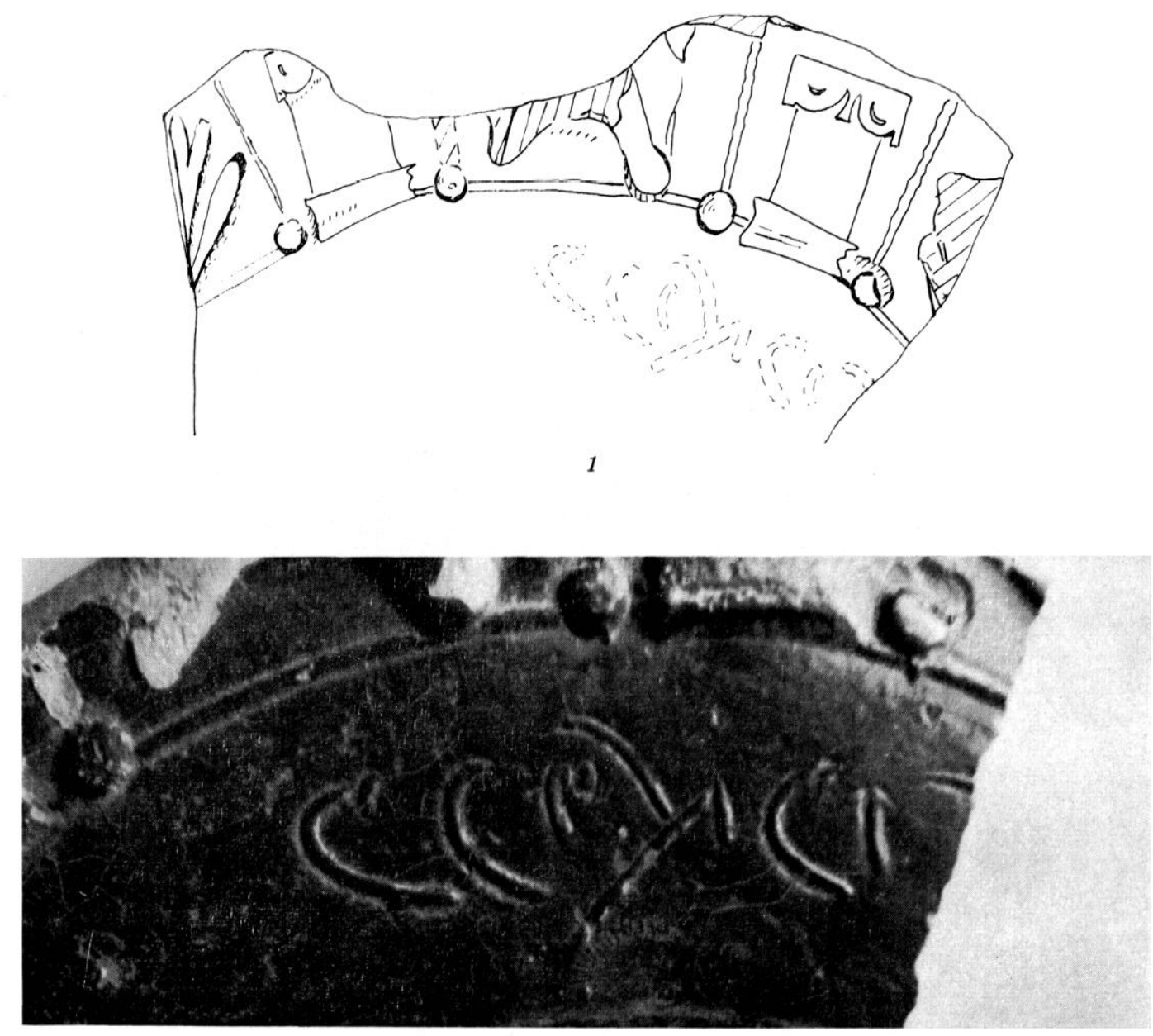

2
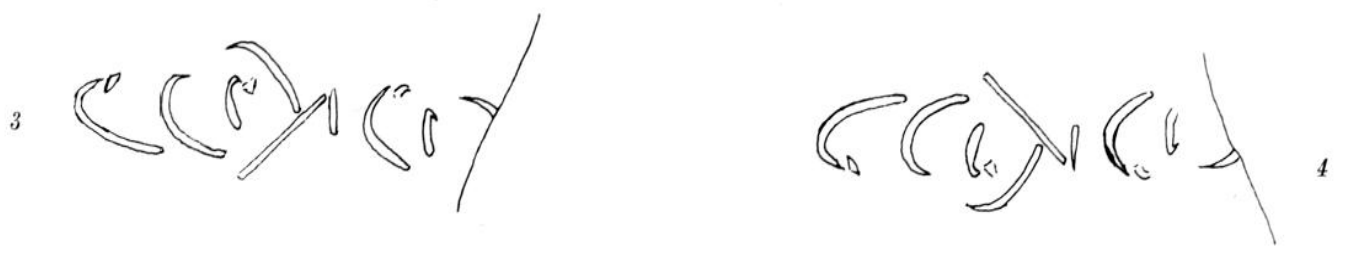

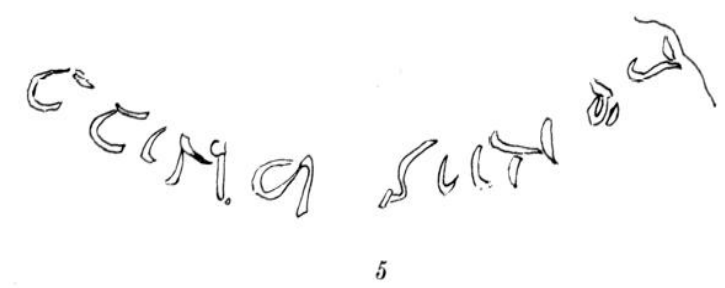

2 Bol c. 1, decor: 2, signature : agrandissement éch. $1 \times 2 ; 3$, signalure : fac-similé telle qu'on la volt); 4, signalure : fac-simile telle fu'plle itait dans le moule). - Bol B. 5 , fac-similé de la signature telle qu'elle etait dans le moulei. 
C. CINO qui soit en bon état : celle du premier fragment de Saint-Rémy ${ }^{6}$. Cela étant, il importe loutefois de remarquer que le type des lettres employées est, chaque fois, le mème et. surtout. que le caractire d'unicité de chaque signature s'accommode de différences notables dans la "mise en page" et la forme des signes. Un doute subsiste cependant, car une sorte de petit trait d'arrêt, à l'extrémité inférieure de la courbe de l'O, fait hésiter sur la lecture de cette lettre. A l'examen, il peul s'agir d'un défaut (comme celui que présente l'l) tout aussi bien que d'un tracé délibéré. line fois ces réserves faites, il reste qu'il est tout à fait possible de reconnaître $C$. CINO el de considérer la ligne interrompue qui suit l'O comme l'extrémité de la courbe inférieure d'un $\mathbf{S}^{7}$. Ainsi, on ne saurait tirer argument de simililudes frappantes pour attribuer cetle signalure incomplete à C. CIVO SEVOI - - , mais il n'existe pas d'obstacles majeurs à une lelle identification.

La deuxieme marque (fig. $4,2,3,4)^{8}$, qui provient de Cavaillon, est très fruste et la pâte du vase, mal tassée, présente des accidents qui défigurent encore les lettres. On lit, cependant, sans grandes difficultés, C. CINO SEN :- - ].

La troisicme (fig. $3,2,3,4)^{9}$, la plus intéressante, est amputée en son début dont il ne reste que - - - и]0. Mais, au-delà du fragment du troisième élément que nous possédons déjà : $S E \mathrm{CV}^{\prime}$, l'inscription continue. Elle est, il est vrai, très empàtée el assez peu distincte. Cependant, on reconnaît, sous un éclairage convenable, la partie inférieure d'un I, puis un $\mathrm{R}$ complet. A la suite, on pourrait supposer une haste légèrement inclinée (fig. 3,3 et 5$)^{\mathbf{1 0}}$,

6 Le's signatures sur les trois autres tessons sont trop floues, ou délériorées, puur qu'on puisse juger avec sécurite de l'aspect des caracteres employés. Ceux-ci, néanmoins, semblent assez proches des lettres de la premiere inscription de Saint-Rémy. Cf. fig., 3, 2, 3, 4: 4, 2, 3, 4 el (iallia, XXV'1, 1968, pl. 11, 2-5.

7 M. R. Marichal, que j'ai consulté à co sujet, tient cette lecture pour la plus satisfaisante.

$x$ Kéf. 55-12-1. Le tesson, trowvé fortuitement en surface, ne fournit aucun indice chronologique.

9 Saint-Rémy, no 291, ancien fonds.

10 Cette trace, accentuée par la photographie, se trouve, en fait, comprise dans une série de creux et de bosses dont le dessin donne une idée un peu plus exacte. dont il subsisterait un morceau. Mais les défauts du lissage ne permettent pas d'assurer qu'il ne s'agit pas là d'un simple accident. Donc le troisieme nom de cette signature devait être SENOVIR ou SEVOVIROS"r. Il est possible qu'on ait ici une forme abrégée. Il faudrait arlmettre, alors, qu'il n'y a, après le K. qu'un tracé accidentel. Gar l'aspect de relle sorte de hasle pourrait, sans doule. évoquer un $\mathrm{F}^{12}$. mais l'absence de prolongement sous la ligne et de barres transversales, en mème lemps que l'intervalle entre cette lettre et le $\mathrm{R}$ (alors que l'espace entre [cin? 0 et SENOVIR est largement ménagé), rend l'hypothise peu vraisemblable. Il est loisible, également, de supposer, à cette place, la présence d'un I. Mais cetle solution, sans présenter les mèmes difficultés que la précédente. manque de garanties incontestables et de la présomplion favorable qu'auraient fait naître des exemples analogues. Linsi l'on a, gràce à ce fragment, la quasi-certitude que le mot jusqu'à maintenant incomplet était SENOVIR(OS), par ailleurs plusieurs fois attesté $^{13}$, sans, pour autant, connaître la nature des rapports grammaticaux qui l'unissent à C. CINO. Il est donc impossible de se fonder sur cette inscription pour en tirer le moindre argument relatif au nombre ou ì la qualité du ou des hommes ainsi désignés.

Les noms, par eux-mèmes, offrent-ils quelque ressource? On connait, actuellement, deux marques, à Orange ${ }^{14}$, attribuées à un

11 La forme $\$ E N O V I R O S$, la plus normale, d'un point de vue philologique, est le nominatif aurquel on rapporte $S E N O V I R I$, seul atteste cf. A. HoLbir, Allcellischer Sprachschalz, II, col. 1501, 1-10; K. H. Scumnt, Die Komposilion in gallischen Personennamen, dans Zeilschrifl für cellische Philologie, 26, 1957, p. 267). SENOVIR forme abrege sous l'influence. du latin ?) serait altesté à Nancy. Mais je n'ai pu avoir acces au Calalogue du Musée lorrain cilé par A. Holder, ni connaitre le contexte de cette inscription.

12 Abréviation de fecil ou fecerunl. Le verbe, bien que plus rare dans les signatures manuscrites externes, se rencontre cependant (cf. le moule de liheinzabern publić par Linowici, I, p. 107 : SIIVIlliANVS GEMIILLVS FIICIIIRVNT AMBO).

13 C.I.I. XII, 3584 Nîmes) ; XIII, 4711 (Épinal); XIII, 5569 Dijon); Calalogue du Musée lorrain, no 156 . Nancy).

14 C.I.L., XII, $5656 ; 816$;. 


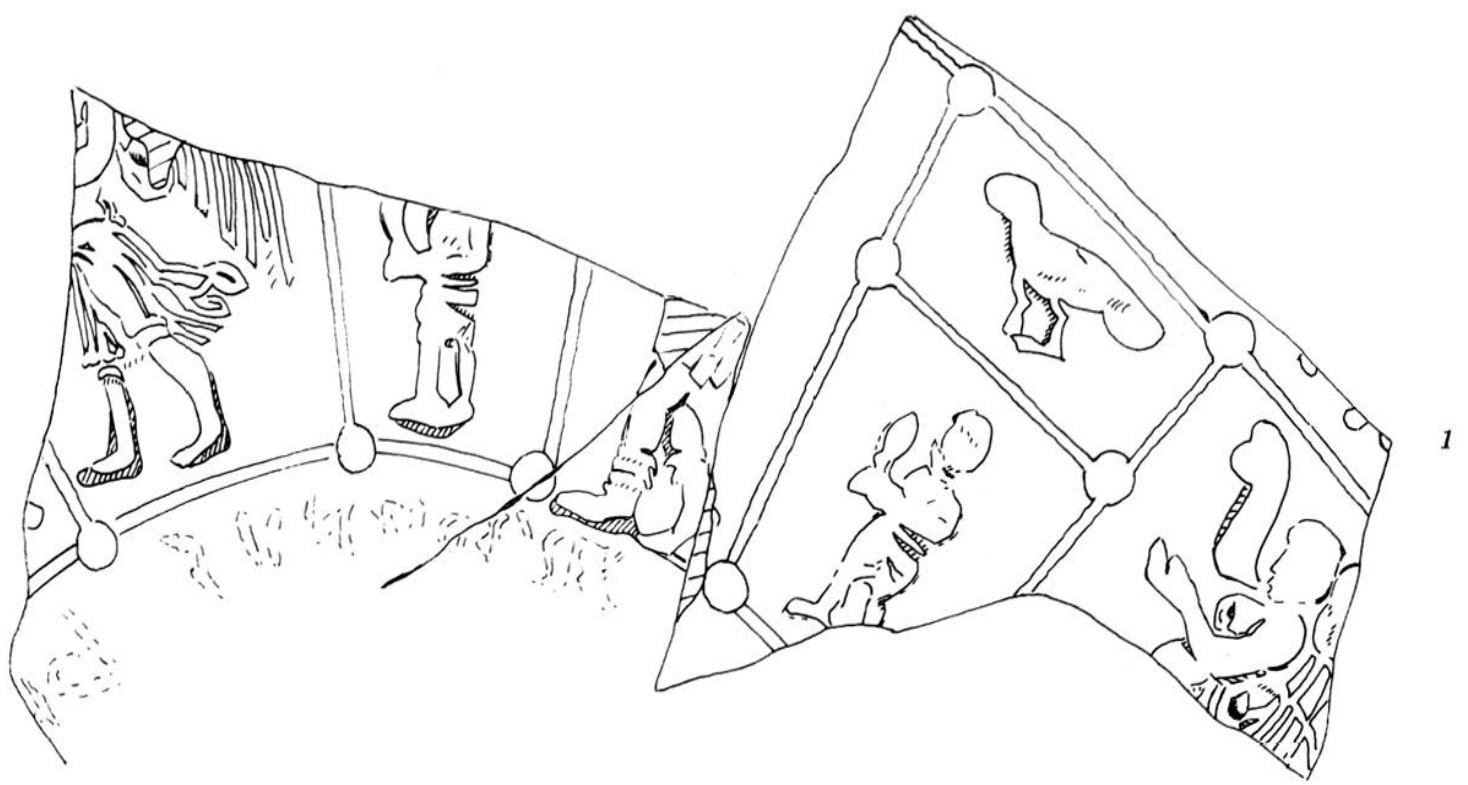

3<smiles>CCCCC=C(C)C</smiles>

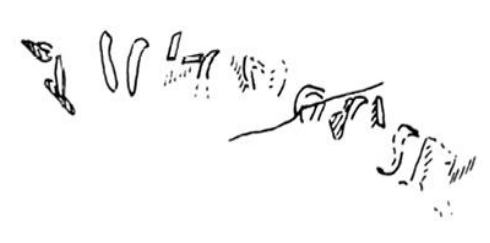<smiles>Cc1ccccc1CCc1ccccc1</smiles>

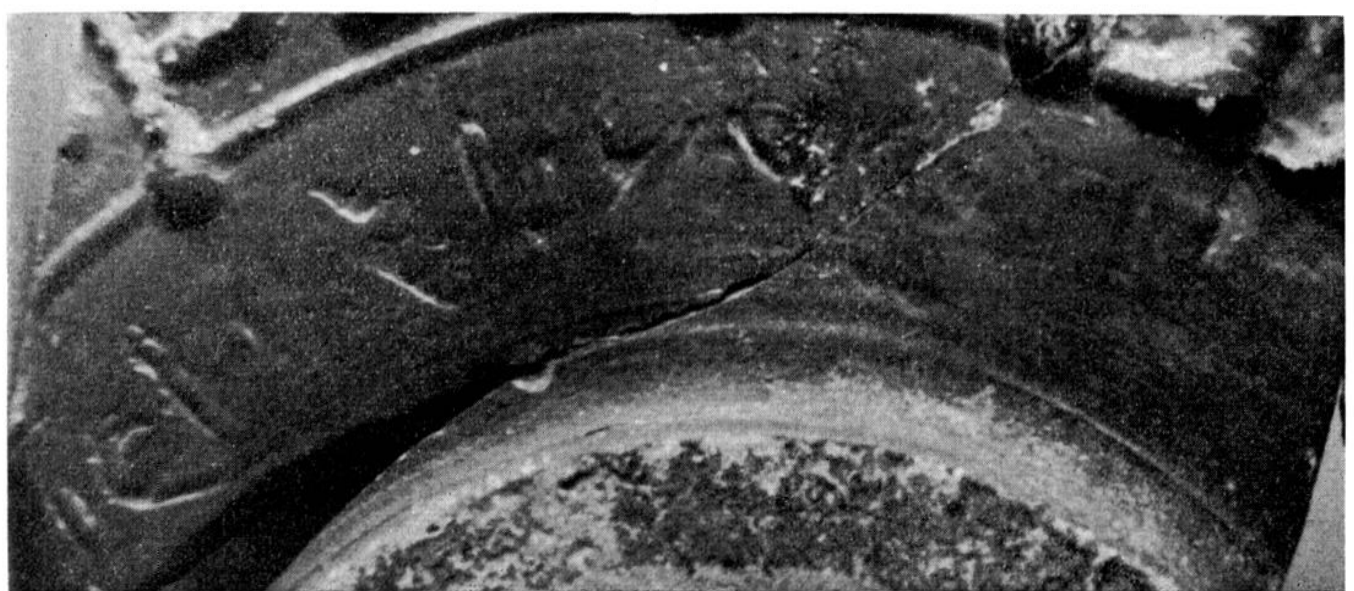

3 Bol D. 1, décor; 2, signature : photographie taille réelle); 3, signature : fac-similé telle qu'on la voit); 4, signature : fac-simile telle qu'elle stait dans le moule); 5 , signature : agrandissement éch. : $1 \times 2$ ). 

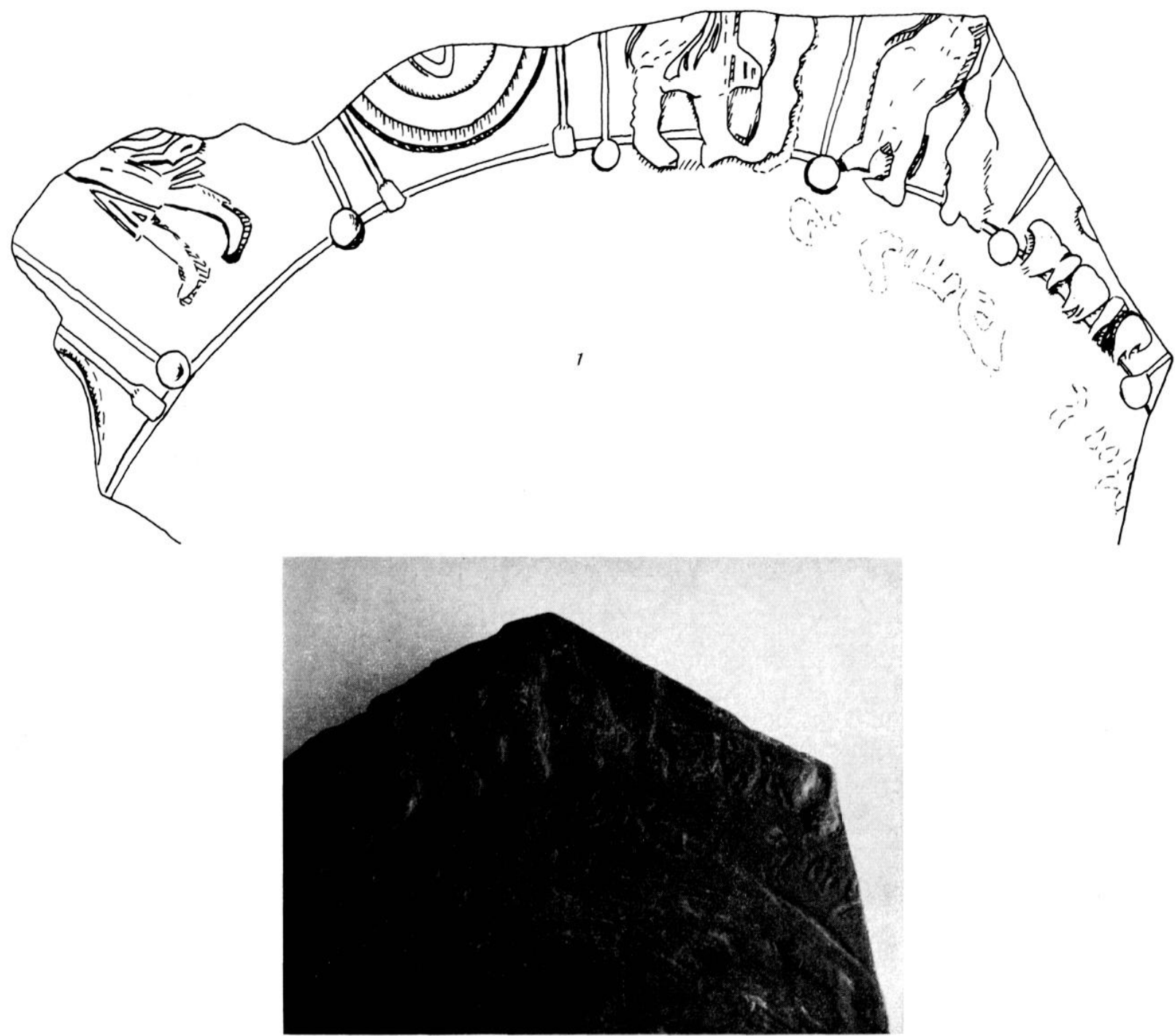

2

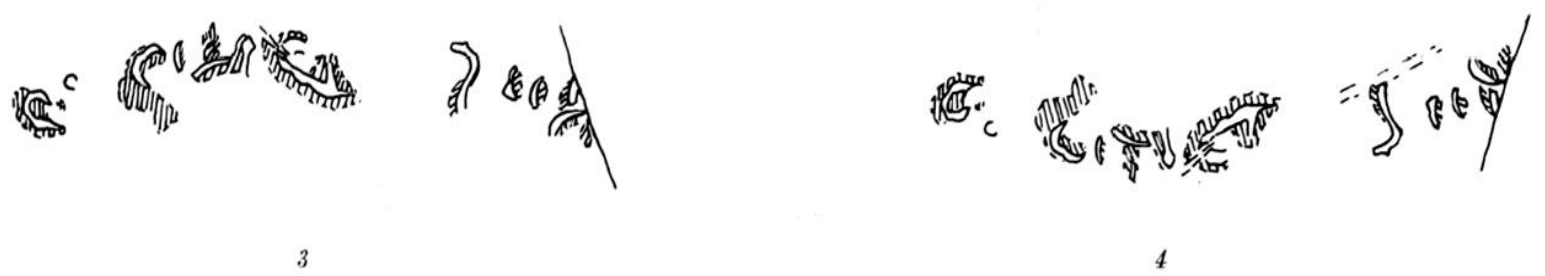

4 Bol E. 1, décor; 2, signature : photographie (taille réelle); 3, signature : fac-similé (telle qu'on la voit); 4, signature : fac-simile telle qu'elle était dans le moule). 
SENOVRVS par l'éditeur du C. I. L. XII ${ }^{15}$, aussi bien que par A. Holder ${ }^{16}$. La signature se présente, dans les deux cas, sous la forme OF $2 E$ YORI. La facilité avec laquelle, dans les estampilles gallo-romaines, on confond, sans indiquer la ligature, l'I avec la haste de la lettre qui le précède ou le suit ${ }^{17}$, autorise à croire que l'on peut aussi bien avoir affaire a un SEYOYIROS ou SENOVIRVS ${ }^{18}$. Yais ce potier a échappé au recensement des grands catalogues ${ }^{19}$, et ce fait nous prive de renseignements sur le lieu et l'époque où il exerçait son activité. Il est done exclu, au moins provisoirement, que l'on établisse entre lui et le SENOVIR(OS) de Cavaillon et Saint-Rémy un rapport autre que de possible homonymie. En ce qui concerne l'autre partie de l'inscription. C. $C I N O$ demeure inconnu parmi les noms de potiers actuellement publiés. II. II. Vertet signale, dans un article récent ${ }^{20}$, une marque trouvée à Lezoux, dans un dépotoir de l'époque de Tibère, et présentant la forme СІИО[-- -]. Il s'agit d'une estampille incomplète et l'on ne sait quelle était la forme exacte de la signature. De plus, l'absence de prénom, la date ${ }^{21}$ inciteraient, à elles seules - $\dot{a}$ supposer

15) Ibid., p. 900, Index.

16 Op. cil., col. $1500,53-54$.

17 L'étude de plusieurs collections, en particulier de celle de Saint-Rémy, m'a permis de remarquer souvent ce que je considere comme une ligature invisible, plutiot que comme une ellipse. Chaque fois, en effet, que l'on constate l'omission de l'I par comparaison avec des formes plus completes), le tracé d'un caractere immédiatement voisin comporte une haste, verticale ou oblique, et rend aisée la liaison avec cette lettre (Cf. op. cil. Gallia, XXVI, n. 41).

18 sous deux réserves toutefois : il faut que la lecture soit exacte (or le C.I.I. corrige la premiere interprétation 2ENQVI); il n'est pas absolument exclu qu'il puisse s'agir, ainsi que me l'a suggérí .. L. Balsan, de la marque d'une association inédite : SENO et VIRILIS, par exemple.

19 A. Lombard-Dumas (Memoire sur la céramique antique dans la vallée du Rhône, Nîmes, 1879, pl. 6, 153) donne un bon fac-similé, une lecture critique (S rétrograde, O douteux) mais sans référence utilisable.

20 Céramique sigillée Tibérienne à Lezoux, dans Rev. Arch., 1967, 2, p. 255-286, surtout p. 258, fig. 1, 24 et p. 259. .1. Vertet, très prudent pour l'attribution d'une marque dont ce paraît être le premier exemplaire connu, ne lui consacre aucun commentaire particulier.

21 Puisque la forme même de nos tessons, indípen- que CINO fût le nom de l'artisan de Lezoux -..., à n'envisager qu'avec la plus grande prudence l'éventualité de rapports entre CINO $\ldots$ et C. CINO. Il est donc impossible, pour le moment, d'établir aucune parenté entre les éléments de notre signature et des noms de potiers par ailleurs connus et, en conséquence, de situer C. CISO SEYO IRIR..., par ce moyen, dans un milieu professionnel. Néanmoins, si l'on veut épuiser les ressources qu'offrent les inscriptions de ces vases, on doit noter une curiosité : parmi les exemples recensés du nom SENOVIR(OS), trois proviennent du NordEst de la Gaule (Épinal, Nancy, Dijon) et le quatrième de Nîmes22. On connaît de CINO deux emplois : l'un à Eisenberg, dans le Palatinat, oil LVCIOS fils de CINO fait une dédicare à Sillanus ${ }^{23}$, l'autre à Rome. Il s'agit, alors, d'une inscription du ${ }_{111^{\mathrm{e}}}$ s. comportant le nom d'un Y. COTIVS CINO $O^{24}$. Le gentilice parait celtique ${ }^{25}$. Mais l'origine exacte de $M$. COTIVS CINO est inconnue. Le nombre des attestations est trop faible, leur chronologie trop imprécise pour qu'il soit possible de tirer des déductions de ces faits. Néanmoins, il est permis de noter la coïncidence : les deux noms

damment de la nature du décor, implique une datation tardive, sans que rien, pour l'instant, vienne combler l'intervalle qui sépare la fabrication du vase de Lezoux de celle des bols de Saint-Rémy et de Cavaillon. D'autre part, la distance géographique entre Lezoux et l'atelier du Sud de la Gaule auquel on pouvait déjà attribuer nos premicrs fragments peut constituer un obstacle supplémentaire, en la circonstance. Elle ne saurait, cependant, s'opposer, à elle seule, à l'hypothèse d'une éventuelle identification, tant on connait actucllement de potiers altestés, à la même éporque ou successivement, dans deux ou plusieurs centres de production.

22. Épinal : SEX. I IVV SENOVIRI/DVBNOTALI F./IVL . LITVIIARA. LITAVICCI , F/MATER, FACIE NDVM/CVKAVIT I $^{\text {er }}$ s. p.C. ?).

Nancy : SENOVIR.

Dijon : SEN. - - ? /SENOVIRI/ - . ? VIRI

maintenant disparue).

Nîmes : FIRMINO/SENOVIRI/F.

23 C.I.L. XIII, 6146.

24 C.I.L. V1, 1058, en 210.

25 Cf. A. Houner, op. cil., I, col. 1143, 12-21. Les autres COTII connus figurent au C.I.L. III et $V$, à l'exception d'un seul, qui, attesté à Rome, est dit originaire de Mesie cf. C.I.L. VI, 2933. 
de la signature sont celtiques ${ }^{26}$ et, quels que soient les rapports entre eux, ils ont des chances d'avoir été employés surtout, l'un, dans le Nord-Est de la Gaule, l'autre, aux confins de la Germanie, ou d'être originaires de ces régions ${ }^{27}$.
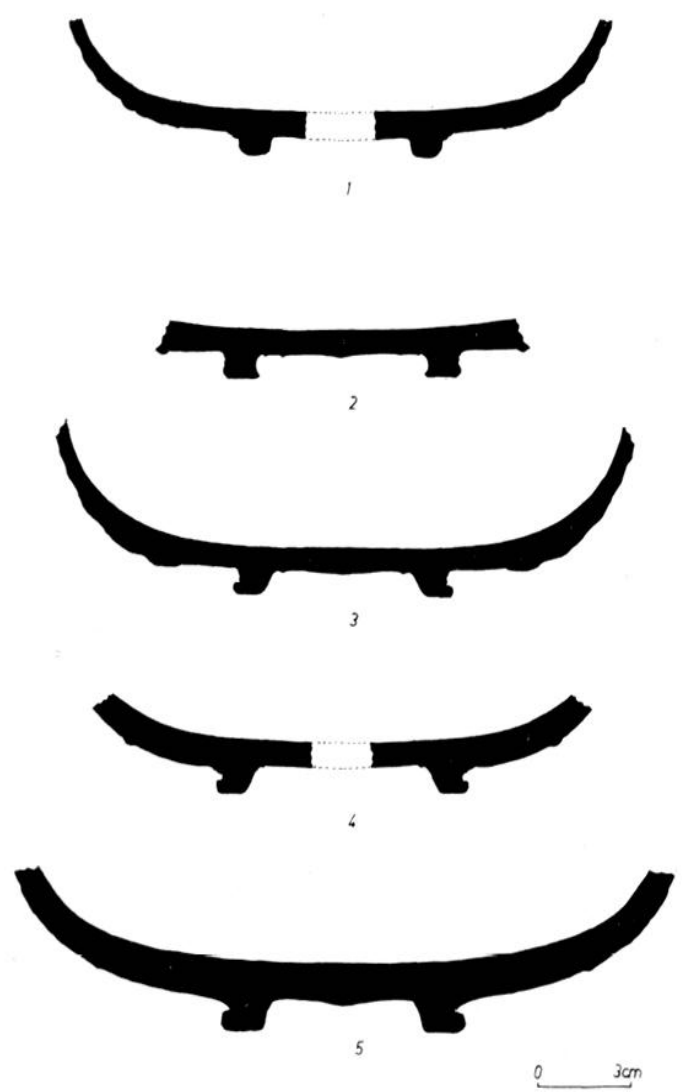

5 Profils. 1 , bol B ; 2, bol A; 3, bol I) ; 4, bol C; 5 , bol E.

L'identité de C. CINO SENOVIR... n'apprend, en définitive, qu'assez peu de choses. Si l'enquête autour de cet atelier doit être étendue, elle ne saurait l'être, désormais, que par l'étude des vases sur lesquels figurent ces signatures, et des moules d'où ces bols sont issus. Comme l'analyse de l'argile est l'un des moyens les meilleurs -..- même s'il est imparfait ... de déterminer l'origine d'une

26 Voir, pour plus de détails, mon précédent article (loc. cit. p. 304-306).

27 Si d'autres marques permettaient de supposer des rapports de filiation entre CINO et SENOVIR, la coincidence pourrait paraître moins fortuite. céramique $^{28}$, j'ai demandé à II. A. Blanc ${ }^{29}$ d'examiner ces nouveaux fragments. I a terre est, dans deux des $\operatorname{cas}^{30}$, semblable à l'argile insolite du premier tesson analysé de C.CI.YO $O^{31}$. Riche en chaux, elle se distingue de la pâte du troisiome fragment : celui de Cavaillon, qui présente, elle, toutes les caractéristiques des fabrications tardives. Mais, quel que soit le dosage de la chaux, les trois exemples, si on les compare aux échantillons actuellement recensés à Valence, présentent les plus grandes analogies avec l'argile utilisée à la ciraufesenque ${ }^{32}$.

L'examen des trois tessons (fig. 1) ${ }^{33}$ révèle, du point de vue de la forme, des différences notables de l'un à l'autre (fig. 5, 3, 4, 5) : E. trìs épais et tris lourd, devait, d'après le fragment qui subsiste, ètre un grand bol, assez largement évasé; son profil, plus bas que celui de $\mathrm{C}$, est loin, cependant, de la courbure brutale de 1). Les pieds, de diamètre extérieur variable ${ }^{34}$, mais de hauteur à peu près identique dans les trois cas ${ }^{35}$, se distinguent aussi par la gorge qui les allege sous la panse et par la largeur de leur assise inférieure : la gorge est sensiblement plus haute et profonde pour $\mathrm{C}$ et $\mathrm{D}$, l'assise, plus étroile ${ }^{36}$; le pied du bol E est massif, ce qui peul s'expliquer par des raisons fonctionnelles : $\mathbf{E}$, épais, large et lourd, se serait mal accommodé d'un pied gracile. Cependant, les trois vases présentent des caractéristiques communes qui les distinguent de B (fig. $\overline{5}, 1$ ) : le dessous du pied forme

$28 \quad J$ 'ai constate la valeur de ce procéde, encore au stade de l'experimentation, pour la localisation de céramiques unies estampillées du Musée de Saint-Rèmyde-Provence. I. 'origine proposie s'est trouvée confirmie, a posteriori, par la presence de ces marques parmi les inédits de la Graufesenque.

29 Qui fait des recherches aul Iaboratoire de céramologie de Valence.

30 Nos 291 et 3064.

31 Op. cil., p. 308-309.

32 Elle se distingue, en particulier, des argiles de Banassac jusqu’à présent connues.

33 Par commodité, je les désigne ainsi : fragments publiés précédemment : Cavaillon $=A ;$ Saint-Rémy $=$ B; nouveaux tessons : Saint-Remy no $3064=\mathrm{C}$; $\mathrm{n}^{\circ} 291=\mathrm{D}$; Cavaillon $55-12-4=\mathrm{E}$.

$34 \mathrm{C}: 82 \mathrm{~mm} ; \mathrm{D}: 72 \mathrm{~mm} ; \mathrm{E}: 80 \mathrm{~mm}$, tandis que A mesure $76 \mathrm{~mm}, \mathrm{~B}, 66 \mathrm{~mm}$.

$35 \mathrm{C}: 8 \mathrm{~mm} ; \mathrm{D}: 7, \overline{5} \mathrm{~mm} ; \mathrm{E}: 8, \overline{5} \mathrm{~mm}$.

$368 \mathrm{~mm}$ pour C et $\mathrm{I}, 10 \mathrm{~mm}$ pour E. 
une couronne très plate, qui permet un contact sur presque toute sa largeur avec la surface sur laquelle on le pose; la base comporte, dans les trois cas, un bourrelet arrondi, nettement saillant vers l'extérieur. Le profil de $\Lambda$, en revanche, est proche de celui de $\mathrm{C}$ et de D : par le diamètre extérieur du pied, son assise large et plate, sa gorge haute et moulurée. De légères différences apparaissent cependant : bourrelet peu saillant, profil intérieur concave. Mais il faut noter aussi, malgré la faible surface du fragment conservé et l'absence de tout renseignement sur la forme de la panse, une autre analogie avec D) : un fond remarquablement plat, jusqu'à la limite du décor (fig. $5,2,3$ ). Au-delà de ces constatations, il me serait impossible, toutefois, de déterminer avec certitude si les variations dans le profil des moules - d'ailleurs trop incomplets pour la plupart - sont liées à une évotution chronologique de C. CINO, ou si les modifications dans la forme du pied dépendent de la date plutôt que de l'ouvrier chargé des finitions, ou, encore, du module du vase ${ }^{37}$.

En ce qui concerne la technique du décor (c'est-à-dire la fabrication de la matrice), on voit que, sur les bols $\mathrm{C}, \mathrm{D}, \mathrm{E}$, comme sur $\mathrm{B}$ et $A$, la zone ornée s'arrête sur une simple ligne en relief (fig. 2,$1 ; 3,1 ; 4,1.6,2)^{38}$. Comme sur B - et vraisemblablement sur A, ainsi que tend à le faire croire une rosette d'angle conservée -... le système d'occupation de la surface repose sur l'emploi des métopes, avec une alternance entre les panneaux pleins et coupés ${ }^{33}$. Le schéma peut varier d'un vase à

37 En ce qui concerne la largeur du pied, on peut remarquer que les bols dont les formes présentent le plus d'analogies - du moins dans la partie inférieure de la panse -, c'est-à-dire $A$ et $D$, d'une part, et C: et $E$, de l'aulre, diffèrent peu par la dimension de leur base. $B$, qui ne s'apparente de pres à aucun des autres, a le pied le plus étroit. On constate, par ailleurs, que le corps de ce vase a, à la fois, le plus petit diamètre de ceux qu'on peut imaginer pour l'ensemble de la série et, la plus faible épaisseur.

38 Le tesson A, presque totalement privé de décor, porte cependant un tout petit fragment de ce cercle en relief.

39 Celte division est incontestable pour D. En C, il subsiste un bref morceau de cordon au-dessus de l'autel de droite et la séparation se rétablit logiquement au-dessus de la touffe d'herbe, d'apris le modèle de $B$. l'autre, mais la petite étendue des fragments conservés ne permet de reconstituer complètement, avec certitude, ni le développement circulaire d'un décor ${ }^{40}$, ni la succession verticale des métopes. Néanmoins, la présence, sur chacun des quatre tessons décorés $(\mathrm{B}, \mathrm{C}, \mathrm{D}, \mathrm{E})$, d'un motif, au moins, entouré de deux figures identiques m'a amenée à envisager l'hypothèse de l'existence, dans l'atelier de C. CINO, d'un ou de plusieurs systèmes de composition. En examinant d'autres vases, j'ai constaté que bon nombre d'entre eux présentaient une disposition des surfaces pleines, coupées ou recoupées autour de deux diamètres perpendiculaires de la couronne idéale, qui correspond au développement à plat du décor. Ces lignes servaient d'axes de symétrie et passaient, chacune, par le milieu d'un panneau. A l'intérieur de ces cadres, on pouvait distinguer différents nıodes de répartition des métopes autour de la circonférence : en particulier, la répétition du même panneau à égale distance de part et d'autre d'un axe, ou la reproduction de la mème séquence, toujours dans le même ordre, dans chaque quart de cercle.

En second lieu, il m'est apparu que, dans certains cas, à la symétrie géométrique de l'aménagement de l'espace à décorer s'ajoutait soit la répétition du même motif à la même place, soit l'utilisation, à ce même endroit, d'éléments de décor semblables mais opposés par leur orientation. Pour rendre mon propos plus clair, je reproduis ici les fragments d'un vase, sans signature, de Vaison-la-Romaine (fig. 6, 1) : des panneaux analogues se répondent de part et d'autre des axes et à égale distance de ceux-ci; on observe également une corres-

Pour E, la confrontation de nos fragments de motifs avec des analogues intacts révèle l'existence obligatoire de plusieurs niveaux dans le cloisonnement vertical el peut donner une idée approximative de la hauteur de certaines mótopes : ainsi, d'après les exemples fournis par F. Oswald Index of Figure-lypes on Terra Sigiliata "Samian ware ", Liverpool, 1936-37, nos 103$10 \overline{5}$ ), la Diane de $C I N O$ se range parmi les exemplaires les plus grands; en revanche, le petit Silène, identique, ou peu s'en faut, à celui que publie J. Déchelette (Les vases céramiques ornés de la Gaule romaine, Paris, $\left.1904, n^{\circ} 324\right)$, est de taille beaucoup plus modeste (cf. fig. 8 , nos $13 b$ et $14 b$ ).

40 Comme j'ai pu le faire pour B (op. cit., pl. III). 


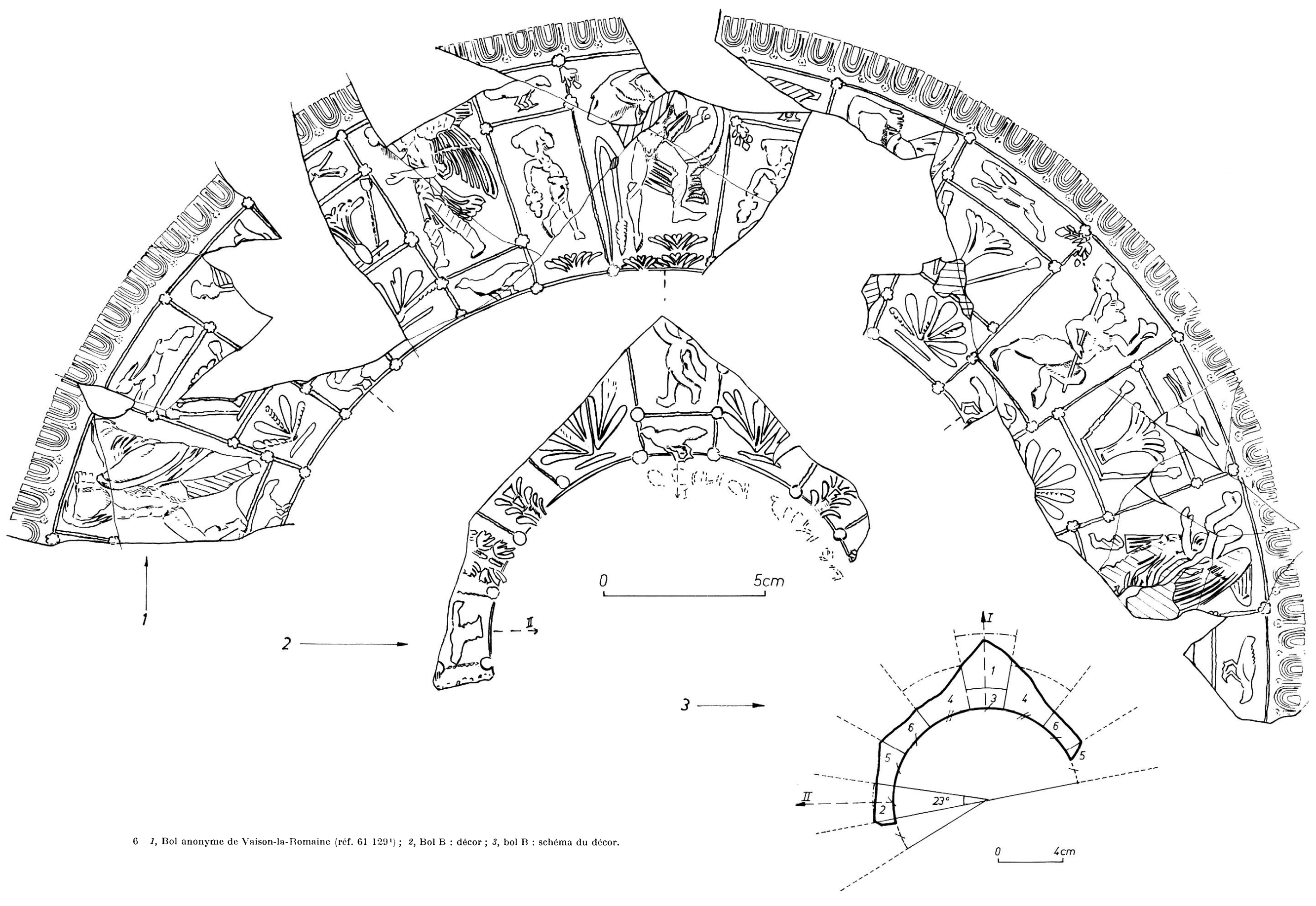




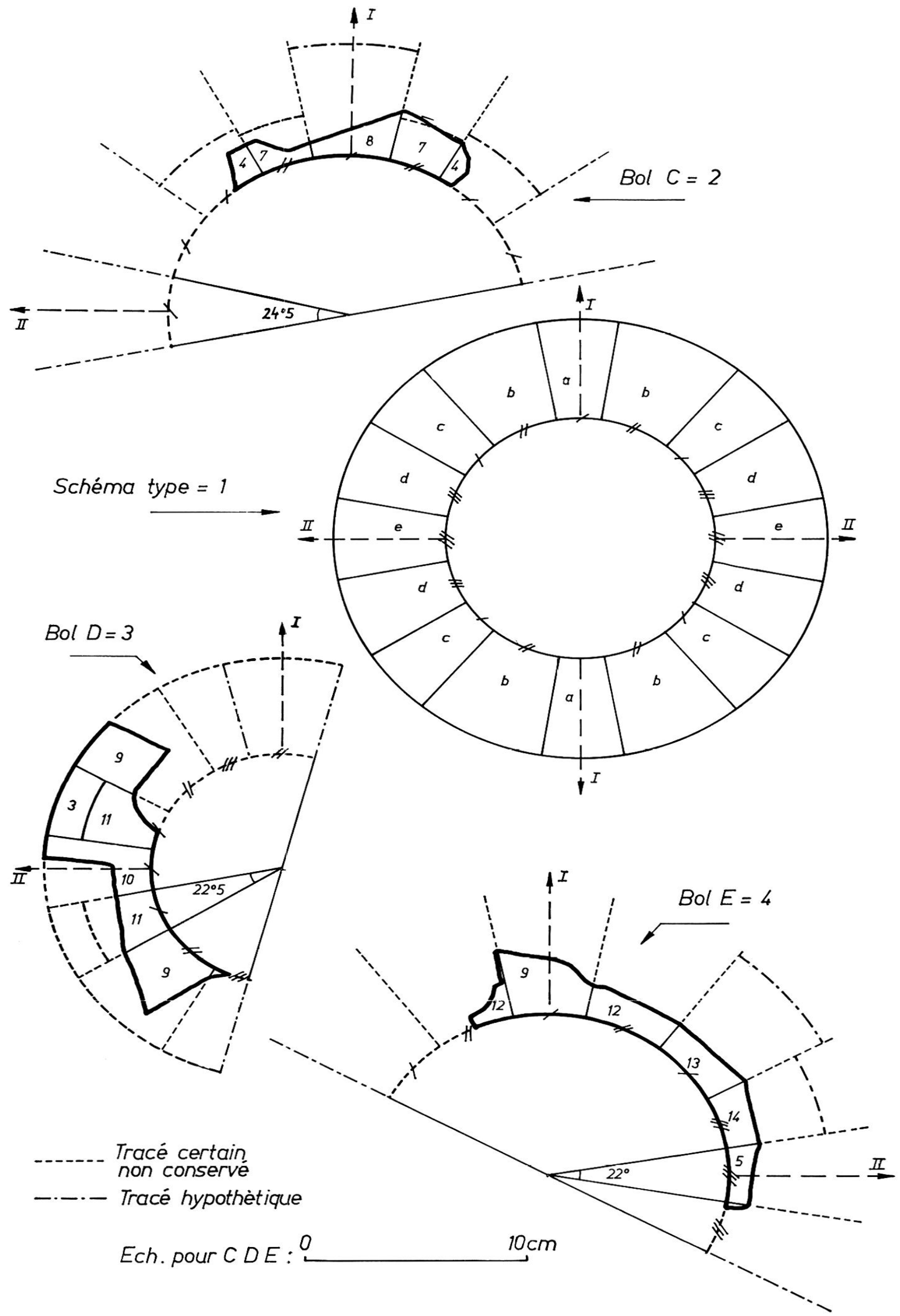

7 Composition des décors. 1, schéma général ; 2, schéma hypothétique de $\mathrm{C} ; 3$, schéma hypothétique de D ; 4, schẻma de $\mathrm{E}$ 
pondance dans les motifs : sans entrer dans le détail de ces combinaisons, il suffit de noter particulièrement la manière dont les axes sont soulignés par la présence, de chaque côté, de poinçons identiques ou afirontés ${ }^{41}$. Or, ces deux aspects - géométrique et décoratif de la symétrie se retrouvent sur les tessons ornés de C. CINO. Il m'a donc semblé possible de considérer ces particularilés comme le reflet d'une habitude du décorateur, à un moment, au moins, de son activité. Et j'ai tenté de voir, d'après les maigres vestiges qui subsistaient, comment il utilisait cette techrique de la composition. D'abord, en étudiant, sur lá circonférence reconstituée de la base de $B$, l'ensemble des divisions à partir desquelles s'est faite la construction du vase, je me suis aperçue que le schéma ainsi obtenu (fig. 6, 3) permettait de faire plusieurs observations : le panneau coupé verticalement par l'un des axes (I) est entouré de deux métopes de dimensions différentes des siennes, alors que sa largeur se retrouve, identique, dans le rectangle qui, de chaque còté, fait suite à celles-ci. En revanche, l'autre axe (II) passe à travers un panneau encadré de deux métopes de largeur égale à la sienne. Or, quand on reproduit, en mesurant soigneusement les distances qui les séparent, les divisions des cercles de base de $\mathrm{C}, \mathrm{D}, \mathrm{E}$, on constate que la répartition de celles-ci permet aux trois tessons de s'intégrer dans le même système géométrique. En effet, E se présente ainsi : le panneau de la Victoire est encadré de deux métopes plus larges, mais retrouve son analogue dans le cadre contenant Iiane (fig. 7,$4 ; 4,1$ ). Puis on rencontre un panneau plus étroit, suivi d'un autre de la même largeur que lui. Le fragment conservé correspond à plus d'un quart de la circonférence. Si l'on admet que l'axe I traverse la métope de la Victoire, on constate alors que la perpendiculaire à celui-ci passe au milieu du second des deux panneaux égaux et contigus. Il apparait, du même coup, que l'on retrouve exactement le type de

41 Identiques et de forme grossirirement sumpitrique : touffes d'herbe; identiques et asymetriques : vendangeurs; semblables et opposés deux à deux : oiseaux. construction du décor de B : même nombre de divisions du quart de cercle, mêmes rapports entre elles autour des axes (cf. Ie schéma général, fig. 7,1$)^{42}$. Sur le fragment $\mathrm{D}$, on peut voir les trois panneaux ${ }^{43}$ conséculifs de mème largeur, encadrés de deux autres, égaux entre eux (fig. 3, 1 ). Ces derniers, compte tenu de la fraction de circonférence conservée et de leurs propres dimensions, pourraient avoir pour réplique la métope de l'axe I, qui nous manque, sans que la division du décor révélée par $\mathrm{B}$ et confirmée par E, se trouve modifiée (fig. 3, 1; 7, 3). Le dernier tesson, $\mathrm{C}^{44}$ n'exclut pas ce système, mais ne le confirme pas absolument. ()n possède la trace de l'axe I : le grand panneau au guerrier entouré de deux métopes plus étroites (fig. 2, 1). Toutefois, ce qui subsiste des deux touffes d'herbe qui viennent ensuite permet seulement de dire que la case qui les contenait avait suirement, au moins, une largeur légèrement inférieure au rectangle du guerrier et, très probablement, une dimension égale ${ }^{45}$. Il est, naturellement, possible d'imaginer, à partir de ce fragment, le reste de la composition (fig. $\bar{i}, 2$ ). Ainsi, à en juger

42 Reconstitué, à partir de ces deux exemples : B donne la disposition d'ensemble et la comparaison montre que les métopes traversées par les deux axes (et les panneaux qui leur répondent) peuvent être soit égales entre elles (B), soit différentes (E). Il importe de préciser que les mesures ont été prises sur les bols eux-mêmes, à l'intersection des divisions verticales et du cercle limitant le bas du décor. Les dessins, obtenus à partir de froltis et pour lesquels je me suis attachée surtout à la reproduction des molifs, faussent les proportions des métopes par la projection a plat d'une surface courbe. Aussi ne devra-t-on pas s'étonner de constater de petites différences entre des dimensions que la mesure directe révèle comme égales.

43 L'assimilation de cette construction aux deux précédentes se fonde, d'une part, sur l'identité de la base de deux métopes consécutives : celle du porteflambeau et celle du Pélée, de l'autre, sur la présence de deux sujets identiques dans les métopes qui encadrent ce dernier.

44 Cette restitution, comme celle de D, bien que vraisemblable, demeure forcément conjecturale, étant donnée la taille des fragments qui subsistent.

45 La touffe d'herbe, apparemment aussi largement étalée d'après les deux morceaux qui en restent sur ce fragment) que celle du bol $B$, suppose une métope egale à plus du double du fragment conservé à l'extrémité gauche du tesson. Or, celui-ci est de peu inférieur à la moitié du panneau contenant le guerrier. 
d'après la répartition horizontale des décors, il est loisible de croire à une certaine constance dans la manière de $C . C I I \%$. Wais le caractère un peu monotone de la division du cercle de base de ces bols ne parait pas lié à l'ouverture invariable des angles au centre correspondant, dans chaque vase, aux divisions de la circonférence. En effet, les cinq métopes qui se répondent autour de l'axe I témoignent, d'un tesson a l'autre, d'un certain goût de la diversité ${ }^{46}$. Toutefois, dans les exemples conservés, la fantaisie paraît beaucoup moins grande dans l'économie des trois métopes de l'axe II : l'angle correspondant, pour le bol $\mathrm{C}$, à la reconstitution hypothétique d'un de ces panneaux est sensiblement plus ouvert que ses analogues pour les vases $\mathrm{B}, \mathrm{I}, \mathrm{E}$; en revanche, ces derniers ne présentent que des variations insignifiantes d'un bol à l'aulre ${ }^{47}$. Enfin, malgré les différences que je viens de souligner, le choix de la largeur des panneaux semble toujours s'être fait en vertu du mème principe : le groupe des trois métopes égales et la séquence des trois panneaux inégaux de l'axe I sont, l'un comme l'autre, inférieurs à un quart de cercle. L'artisan évitait, de cette façon, de partager par le milieu chacun des secteurs compris entre deux axes de symétrie et surtout de faire coincider cette nouvelle division avec la ligne de séparation entre deux motifs. Il échappait, par là, à une rupture dans l'enchainement des décors.

Quelque intérêt que présentent ces constatations, il faut souligner qu'elles se fondent exclusivement sur des mesures prises le long de la limite inférieure du décor. Le potier devait nécessairement marquer, à ce niveau, des repères avant l'impression des motifs et le quadrillage du vase : aussi ces remarques concernent-elles surtout la technique qui a présidé à la construction de l'ornementation. Ilais il ne faut pas s'attendre a en trouver une exacte vérification dans les observations qu'on

46 Elles sont, selon le vase considéré, tantòt plus larges, tantòt plus étroites que la métope de l'axe. La restitution hypothétique de $\mathrm{C}$ el de $\mathrm{D}$, si mes suppositions sont exactes, montre que C devait, comme B, être construit à partir de panneaux de deux largeurs différentes, D, comme $\mathrm{E}$, sur trois dimensions distinctes.

47 C : $24^{\circ} 5 ; \mathrm{D}: 22^{\circ} \mathrm{j} ; \mathrm{E}: 22^{\circ} ; \mathrm{B}: 23^{\circ}$. pourrait faire sur des panneaux conservés dans toute leur hauteur. En effet, il est exceptionnel que l'artisan, en dessinant les lignes de partage de la zone décorée, suive, sur la panse, une direction rigoureusement conforme aux grands cercles verticaux d'une sphère imaginaire, dont le bol représente la moitié, et cela, soit du fait d'une maladresse, soit par nécessité : lorsque l'envergure du poinçon décoratif risque de dépasser les limites de la métope normalement construite il partir de sa largeur inférieure. Il n'en demeure pas moins que, mème imparfaite dans sa réalisation définitive, l'élaboration de l'ornementation par rapport à des axes de symétrie paraîl bien liée aux habitudes de travail du décorateur. On en verra, sans doute, une confirmation dans le fait que, sur les fragments B, C, D, E, la signature commence au niveau de l'un des axes ou immédiatement avant ${ }^{48}$.

La répartition des surfaces, ou, du moins, des largeurs, qui semble asse\% constante dans les moules de C. CINO, s'accompagne de particularités dans la disposition des poinçons décoratifs. lin effet, la présence des axes est, nous l'avons vu, soulignée - comme pour le vase de Vaison ..-par la répétition, de part et d'autre de ceux-ci, du même poinçon. Mlais, en outre, la même symétrie s'observe dans la métope suivante : celle qui occupe, dans chaque quart de cercle, la position centrale. Ce fait se vérifie pour les trois fragments dont les dimensions et la situation par rapport à I et II permettent de disposer, au moins partiellement, de deux exemplaires de ces panneaux -.. c'esta-dire B, C, D ... et aussi bien pour les grands sujets (la Victoire en D, fig. 3, 1 et 7,3) que pour les motifs accessoires (la guirlande de B; la toufte d'herbe de C, fig. 6,2 et $3 ; 2,1$ et 7,2$)^{49}$. On a donc affaire à une composition entièrement fondée sur la répétition de poinçons identiques, deux à deux, de chaque côté des

$48 \mathrm{C}$ : juste à l'aplomb de l'axe I

B : au début de la métope de l'axe I

E : au début de la métope précédant l'axe II

I) : d'apress la reconstitution) sous la métope de l'axe I.

$49 \mathrm{Cf}$. les correspondances indiquées par le numér 0 des poinçons, fig. 6,3 et $7,2,3,4$, pour B, C, D, E et par des lettres, fig. 7,1 pour le schéma général. 
axes. Cependant ces observations n'ont de valeur que par rapport aux fragments conservés et l'on ne saurait affirmer, sans autre preuve, que sur toute la hauteur de chaque vase on n'a utilisé que la correspondance entre les mêmes figures, orientées dans le même sens (la Victoire, les porte-flambeau, sur D.), ou présentant une forme plus ou moins symétrique (les guirlandes, les touffes d'herbe, les autels, sur C et D; de même les festons de E, du moins selon toute vraisemblance). Il n'est nullement assuré, en effet, que, dans les parties supérieures des bols, $C$. CINO se soit interdit de jouer des moyens employés pour tous les registres du vase de Vaison : poinçons opposés face à face (les oiseaux), séquence se répétant dans le même ordre dans chaque moitié du bol (les groupes : Victoire, Pan, petit guerrier ; chien, lapin). Toutefois, même s'il a su combiner de façon variée, diverses possibilités de symétrie (ce qui, pour l'instant, est une pure supposition), une construction rigide et assez dépourvue d'imagination semble la caractéristique des fragments qui nous sont parvenus.

Ainsi, à s'en tenir à ces termes de comparaison dans la forme, le groupe des tessons de Saint-Rémy et de Cavaillon paraît bien lié. $\mathrm{C}$ se distingue par le tracé de la signature et, dans le décor, par les proportions des trois panneaux identiques de l'axe II, si l'on admet, à titre d'hypothèse, la reconstruction d'apres le schéma des trois autres vases. Cette restitution, toutefois, n'a rien d'impossible par rapport aux principes généraux respectés par $C I N O$; de plus, la disposition parfaitement symétrique des motifs conservés est absolument conforme à une manière que je n'ai, jusqu'à présent, retrouvée dans ses détails sur aucun des bols que j'ai pu examiner. En outre, la forme du pied de $\mathrm{G}$ ne diffère pas sensiblement de celle qu'on observe en D. Enfin, la lecture CINO est, de l'avis d'un spécialiste, la plus vraisemblable. Il parait donc raisonnable d'attribuer $\mathrm{G}$ à C. CINO.

A l'intérieur de ces cadres assez étroits, l'homogénéité de cette petite série se manifeste encore par l'emploi commun de certains motifs pour des vases différents. L'oiseau du fragment $D$, bien que plus fruste dans le détail et provenant, sans doute, d'un poinçon plus usé ou d'une copie sommaire, reproduit exactement, dans la ligne des pattes et la position du corps par rapport à ces dernières, la silhouette d'un des oiseaux de B (fig. 8, 3). L'élément servant à composer la touffe d'herbe dans le décor $\mathrm{G}$ se retrouve sur le hol B. Une guirlande de $\mathrm{B}$, encore, était obtenue par l'impression de la partie inférieure d'une feuille cordiforme et découpée, présentée la pointe en bas : ce motif est utilisé en E, dans la métope de l'axe II. Le décorateur l'a imprimé deux fois, en recouvrant la moitié gauche de l'une des feuilles par la moitié droite de l'autre (fig. 8,$4 ; 4,2 ; 8,5$ ). Enfin, malgré l'état assez piteux du vase de Cavaillon ${ }^{50}$, un relevé soigneux des deux exemplaires de la Victoire -... ceux des fragments $\mathrm{D}$ et $\mathrm{E}$. fait ressortir la similitude des détails (fig. 8, 9) ${ }^{51}$. Ce qui autorise à penser qu'on a employé, non seulement le mème élément décoratif, mais le même poiçon.

Au terme de cet examen, nous avons donc une idée assez précise des caractères communs des tessons identifiés dus à $C$. CINO SENOVIR... La connaissance, maintenant plus étendue, de son répertoire et de sa technique permet, peut-être, de le situer, par comparaison, plus précisément qu'auparavant. Les sujets, sans doute, ne sont pas tous recensés, mais si l'on ne sait déterminer toutes les sources éventuelles de l'inspiration de CINO, ou, du moins, loutes les ressemblances entre le catalogue de ses poinçons et celui d'autres artisans, on peut dresser, pour chaque vase,

50 Les reliefs qui devraient être les plus accentués sont comme niveles par rapport aux autres, les bosses en sont remplacées par des surfaces planes, le fond des plis seul subsiste non leur profondeur). On ne peut guire accuser l'usure du moule ou celle des poinçons, qui aurait été moins sélective, ni penser à un empàtement du moule, théoriquement invraisemblable. Il semble plutòt que le vase ait été mal travaillé et qu'on n'ait pas pris soin de faire pénétrer la terre jusqu'au fond des creux, car un balayage de la surface du bol, avant cuisson, aurait laissé des traces qu'on ne distingue pas ici.

51 Voir, en particulier, la déformation apparente de la jambe gauche et les angles aigus sur lesquels s'achevent les plis flottants de la tunique. Une différence très apparente : la saillie des pieds, sur le bol D, s'explique uniquement par l'inadaptation de la courbure du poinçon au profil abrupt du vase. 


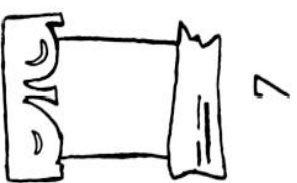

$\frac{5}{8} 6$

$\int_{0}^{3} 5 m$

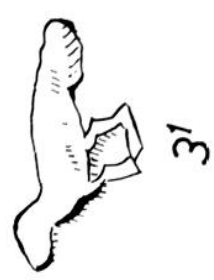<smiles>N#CCCC1CCCCCCC1</smiles><smiles>C1#[Si]C[Si]=C1</smiles>
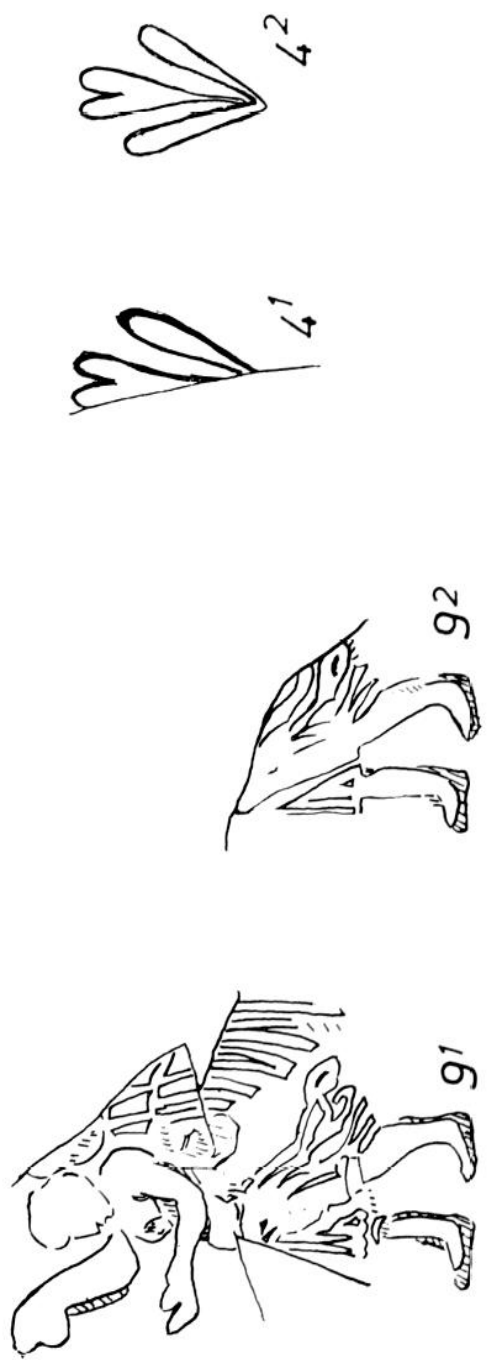

$$
\text { innrion }
$$

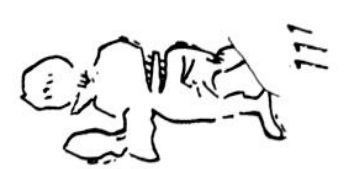
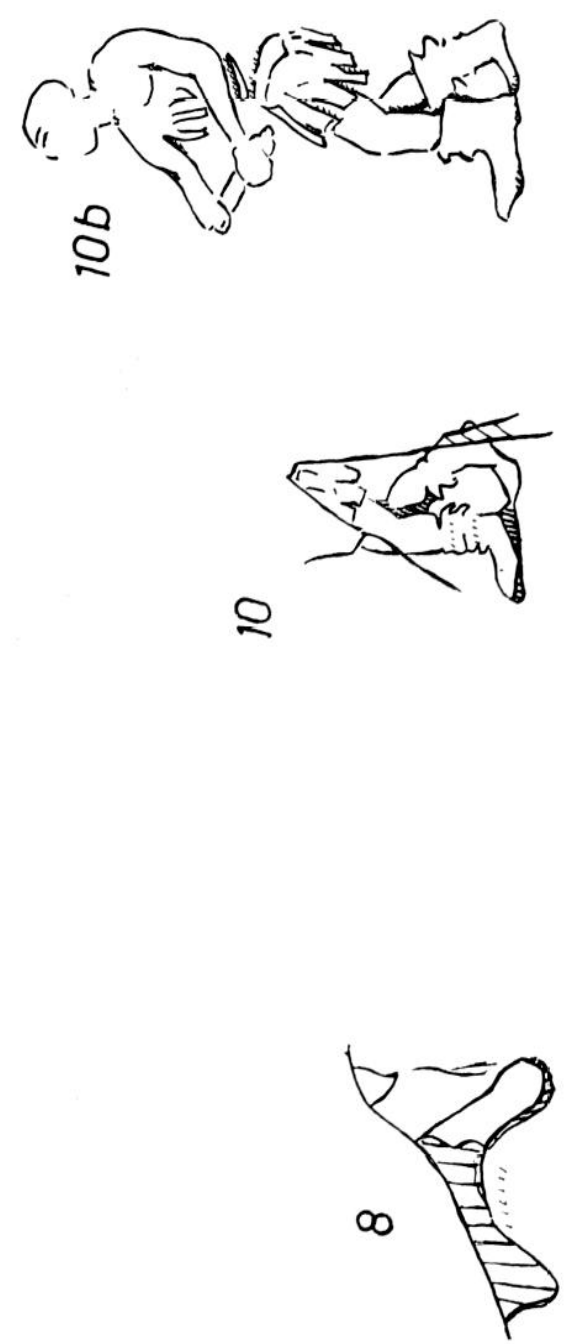

$\stackrel{\infty}{\infty}$

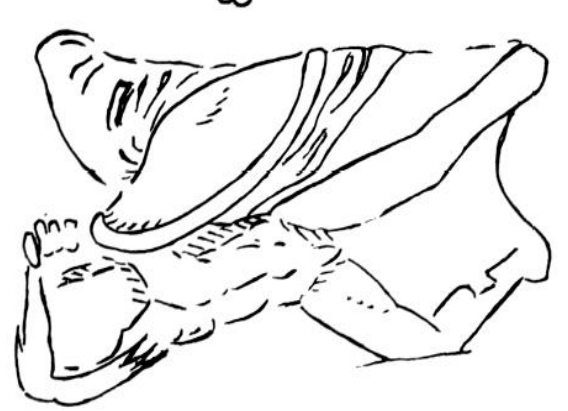



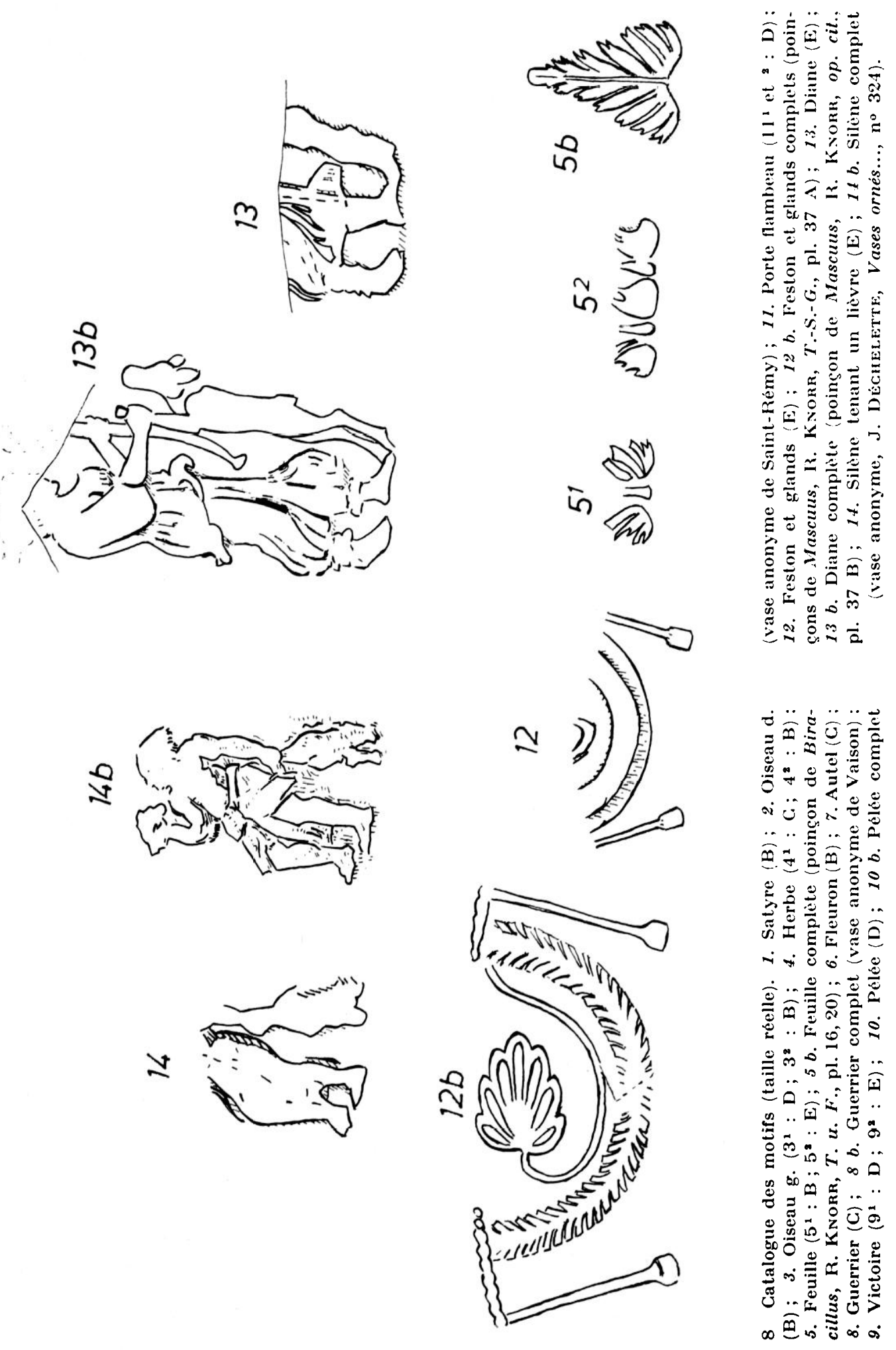
le tableau des motifs et de leurs analogues (cf. p. 203 et fig. 8). La confrontation suggère quelques remarques :

les sujets appartiennent a un répertoire en usage, dans son ensemble, à partir des Flaviens, et, principalement, de Domitien ;

ils ont été utilisés, pour la plupart, soit, d'une façon mal précisée, "à la Graufesenque" et "à Banassac " ${ }^{52}$, soit par des potiers travaillant dans l'un et l'autre centres ${ }^{53}$;

dans la mesure où, d'après les signatures, on connaît les ateliers où furent employés ces éléments décoratifs, les modèles favoris de C. CINO, ou ses émules, peuvent avoir été MASCV $(L) V S^{54}, M E R C A T O R$, voire

52 C'est le cas, principalement, pour une partie des rifirences fournie's par F. Hermet La Ciraufesenque, Paris, 1934, 2 vol.) et. J. Dechelette Vases céramiques...).

53 Ainsi les references donnes par R. Knorr (je renvoie, pour plus de facilité, presque exclusivement à Töpfer und Fabrilien verzierter Terra-Sigillata des ersten .Jahrhunderts, Leipzig, 1919 et Terra-SigillalaGefässe des ersten .Jahrhunderts mil Täpfernamen, Leipzig, 1952) ne permettent pas, le plus souvent, de connaître l'origine géographique des différents produits d'un même polier. Or, même s'il n'est pas exclu que Banassac et la Graufesenque aient pu, avec le Rozier, constituer, à un moment de leur histoire, un complexe unique, rien encore ne permet de confondre ces centres voisins.

54 La question des rapports entre MASCLVS, MASCVLVS, et MASCVVS n'est pas claire. En effet, F. Oswald Index of Pollers' Slamps on Terra Sigillala "Samian uare", Margidunum, 1931) distingue chronologiquement MASCLVS ou MASCVLVS (Claude-Vespasien) el MASCVVS (Domitien), qu'il place tous les deux à la Graufesenque. R. Knorr sépare, lui, MASCLVS de MASCVLVS et il attribue, dans Töpfer und Fabriken (pl. 53), à MASCVLVS un vase publie dans $T .-$.S.- (iefässe (pl. 37 C) avec la marque OF MASCVI. Selon R. Knorr T. $u$. F., p. 19), MASCVIVS aurait travaille a la Graufesenque et a Banassac. Cetle assertion se trouve confirmée, dans un article recent cf. P. Prirk et (h. Horkis, Les exporlalions de ceramiques sigillees de Banassac en Provence et dans les pays Rhodaniens, dans Provence Hislorique, XVIII, fasc. 71, 1968, p. 71), qui signalent l'existence d'estampilles de $M A S C V L V S$ à Banassac. Mais il s'agit de vases lisses dont les auteurs ne fixent. pas la chronologie. Ils ne disent pas non plus s'ils identifient ce MASCVLVS avec le MASCLVS d'Oswald ou avec le MASCVVS/MASCVLVS de Knorr, ou, encore, si, pour eux, MASCLVS et $M A S C V L V S / M A S C V V S$ sont un seul el même potier.
BIRACILLVS, ou L. COSIVS VIRILIS ${ }^{55}$;

la ressemblance avec l'un ou l'autre de ces potiers parait plus ou moins grande, selon le moule de CINO considéré, mais cette apparence ne doit pas nous abuser. En effet, il n'existe pas, actuellement, pour la plupart des centres, de publications étendues des répertoires, atelier par atelier, d'apres, au moins, les vases signés ${ }^{56}$. De plus, et pour la même raison, on ignore la limite des échanges entre les différents fabricants ${ }^{57}$. Aussi, dans le cas qui nous occupe, n'est-il pas exclu que tous les poinçons de CINO apparentent son répertoire à celui d'un seul maitre, mais il n'est pas possible de le prouver, pas plus que de soutenir le contraire;

il n'existe pas, compte tenu de ce qui reste des moules ${ }^{58}$, de petits détails caractéristiques qui permettraient de supposer des conlacts étroils avec un potier, ou un centre, plutôt, qu'un autre. Les oves, en particulier, font totalement défaul.

Au-delà de ce recensement, si je me référe aux comparaisons que j'ai faites, dans l'hypothèse d'emprunts de la part de CINO, on ne peut parler que de modèles. Il est vrai que le choix des motifs, la forme originale que prennent ces derniers suggèrent parfois des ressemblances très précises (fig. 8,13 et $13 \mathrm{~b}$ ) ${ }^{59}$. Cependant, peut-être à cause d'un défaut

5) II. L. Balsan ma confirmé que BIRACILLVS a bien travaille aussi à la Graufesenque :cf. R. Kxonn, T. u. F., p. 18. L. COSIVS VIRILIS est connu à Banassac P. Prire et (h. Morki, loc. cil.).

56 En ce qui concerne les ateliers du Sud, les releves de $\mathrm{I}$. Knorr constituent les instruments les plus precieux, mais ils n'offrent qu'un choix.

57 In exemple remarquable, celui de la ressemblance, confinant à l'identité, d'un décor signé BIRACIL et d'un autre estampillé OF MASCVI (I. Kхони, T.-S.-G., pl. 6 et 37), lend à prouver l'existence de rapports entre ces deux poticrs, sans doute à l'avantage de MASCV(L)VS lein gebender Töpfer, sclon Knorr). Mais on ne sait pas jusqu'où allait colle contamination.

58 En effet, les rosaces, qui soulignent l'intersection de cordons, sont trop usées pour qu'on en reconnaisse la forme originelle.

59 Ainsi les plis de la lunique de la Diane, plus que toutes les variantes publiées par $F$. Oswald Index of Figure-lypes..., nos 103-105), rappellent le poincon de MASCVIVS cf. fig. 8, $13 b$, dapres R. K.оkR, T.-S.-G., pl. 37 c). 
d'information ${ }^{60}$, peut-ĉtre aussi du fait de légères, mais réelles, différences, je n’ai pas trouvé parmi les décors de CINO de poinçons qui montrent indiscutablement qu'il travaillait, à un titre quelconque, dans la dépendance d'un atelier dont il aurait utilisé le matériel (fig. 8, 5, $8,10,12,13,14)$. En revanche, si l'emploi des feuilles cordiformes découpées n'a rien de bien original (même en guirlandes), l'usage qu'en fait C. CINO m'a paru moins banal. Je ne saurais dire s'il lui est propre, mais l'enquête ne m'a pas encore permis de le rencontrer ailleurs.

Quant au style de l'ornementation, les trois nouveaux tessons confirment pour une grande part l'impression que donnait le premier fragment : la sécheresse du décor, d'où sont absentes les feuilles à longues tiges, qui s'accrochent aux angles des métopes, à l'époque flavienne, incite a situer ces compositions plutòl sous le regne de Trajan. Solution qu'invitent également à adopter - si l'on se réfere aux exemples cités par F. Oswald et T. D. Pryce ${ }^{61}$ certains détails, comme l'utilisation de festons isolés que l'on remarque sur le tesson E. Toutefois, l'abondance des motifs végétaux sur le vase $B$, l'utilisation de feuilles et de fleurons en guirlandes verticales, telles qu'on en voit sur les bols flaviens ${ }^{62}$, m'inclinent à penser que les quatre moules peuvent n'ètre pas exactement contemporains et que $\mathrm{D}$ et $\mathrm{E}$ présentent des caractères plus nettement tardifs, dans la mesure où la partie conservée des décors autorise à juger de l'ensemble. Le cas de $\mathrm{G}$ est douteux, étant données les dimensions du fragment conservé. Ce sentiment sur la chronologie relative de nos

60 Outre qu'une masse de documents de référence nous échappent, les relevés ne permettent pas toujours de reconnaitre un poincon en particulier dans le catalogue d'Oswald), ou encore le propos d'un auteur l'amine à choisir un echantillon, qu'il figure aussi précisément que possible et présente, à titre d'exemple d'une série (cf. sur ce point, J. JÉchletette, Vases céramiques..., Introduction au $\mathrm{t}$. II, p. 2 et 3 ). Plutót que de procéder à une restilution nécessairement arbitraire, j'ai préféré suggérer ce que pouvaient être les motifs complets en reproduisant les décors analogues les plus proches que j'aie trouvés cf. fig., $8,5,8,10$, $12,13,14)$.

61 Introduclion to the Study of Terra Sigillata, I.ondres, 1920/1966, pl. XVl et p. 100.

62 Ibid., pl. $\mathrm{XV}, 1$ et 5 . vases a, d'ailleurs, besoin, dans son ensemble, de se confirmer par l'étude d'un matériel qu'on doit souhaiter plus abondant.

Ainsi, ce bref examen permet une connaissance moins hypothétique de l'atclicr de C. CIVO SEVOVIR... : signature plus complète, répertoire plus étendu et mieux circonscrit, localisation probable confirmée, datation précisée. De plus, la technique décorative a pu prêter à un certain nombre de remarques. Mais il reste encore à définir la nature des relations entre les noms $C . C I N O$ et $S E$ YOVIR: la connaissance de ces rapports grammaticaux présente, en effet, un intérêt du point de vue de l'onomastique ${ }^{63}$ aussi bien que des usages dans la pratique artisanale des potiers (à supposcr qu'apparaisse, contrairement aux habitudes les plus répandues, l'indication, dans ces marques, d'une filiation ou d'une dépendance, ou que se révèle une nouvelle association). D'autre part, le catalogue des poinçons de C. CI.VO SEVOVIR... est probablement loin d'être complet il manque, en particulier, des exemples d'oves et il n'est pas possible, faute de fragments assez étendus, de reconstituer complètement les systèmes d'alternance des panneaux pleins ou coupés pour chaque modèle, ni, par conséquent, de relever d'éventuelles constantes dans le détail des principes décoratifs. Il ne serait sans doute pas sans profit, non plus, d'appliquer à des vases signés l'étude des procédés de composition, afin d'étendre et de préciser les comparaisons entre leur manière et celle de notre potier. On ignore, enfin, si $C$. CINO bornait son travail à la fabrication des moules ou s'il produisait, en même temps, des vases, et il manque, quelle que soit la solution à ce problème, une confirmation formelle des hypothèses relatives au lieu où s'exerçait l'une ou l'autrc de ces activités ${ }^{\text {f4 }}$. Seules les découvertes

63 En particulier pour l'utilisation des cognomina indigènes dans les formes romanisées des noms celtiques.

64 Les décors, comme ont bien voulu me le confirmer l'abbé P. Peyre et M. L. Balsan, sont, pour la plupart, très communs tant à Banassac qu'à la Graufesenque et les vases eux-mêmes ont pu être fabriqués dans ce dernier centre. Mais aucune signature semblable n'a êté découverte ni chez les Gabales ni chez les Rutènes. 
de nouvelles fouilles pourront apporter une certitude. Il est probable, toutefois, qu'un fabricant qui a laissé, entre Cavaillon et Saint-Rémy-de-Provence, la trace de cinq moules différents, s'il n'a pas travaillé à proximité immédiate, a dû avoir une production non négligeable. Et s'il n'avait créé, même, que ces cinq modèles, les bols que nous connaissons paraissent, pour certains, issus de formes si usées qu'on peut raisonnablement penser que nombre d'autres vases ont été façonnés à l'aide des mêmes matrices. J'ose donc espérer que de nouvelles trouvailles ou, simplement, l'identification d'autres tessons permettront de parfaire ce travail.

Colette BÉмonт. 


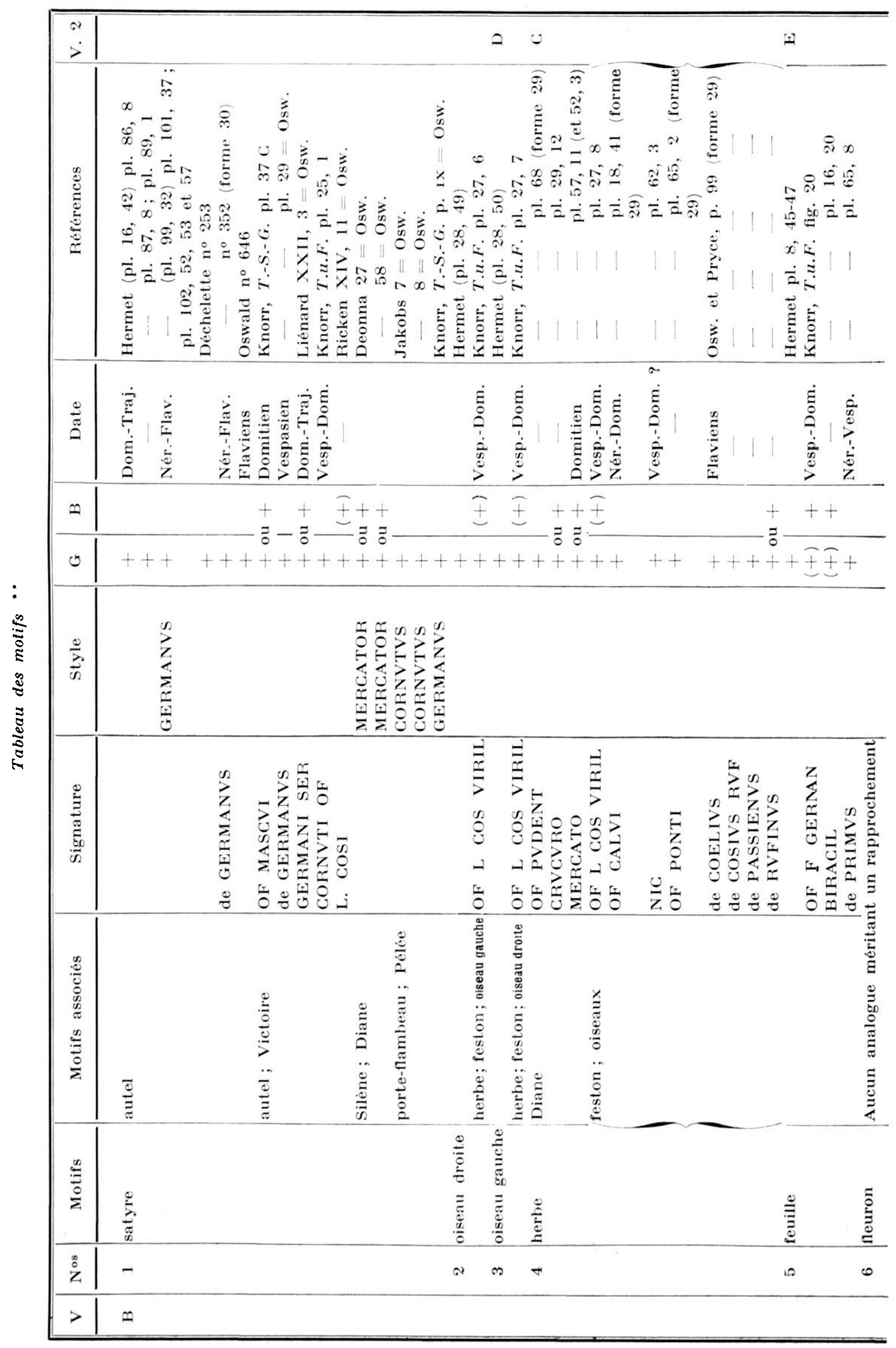




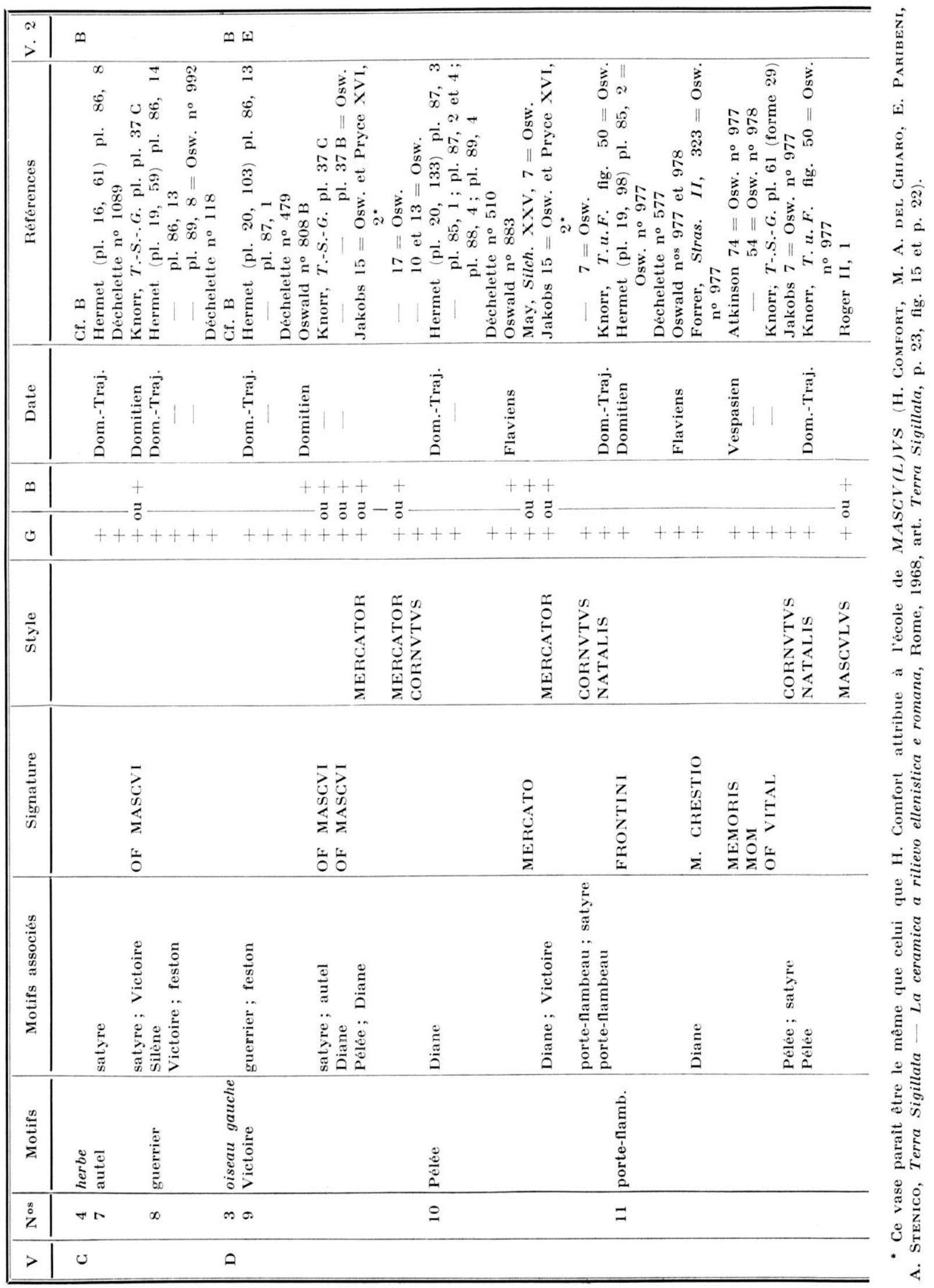




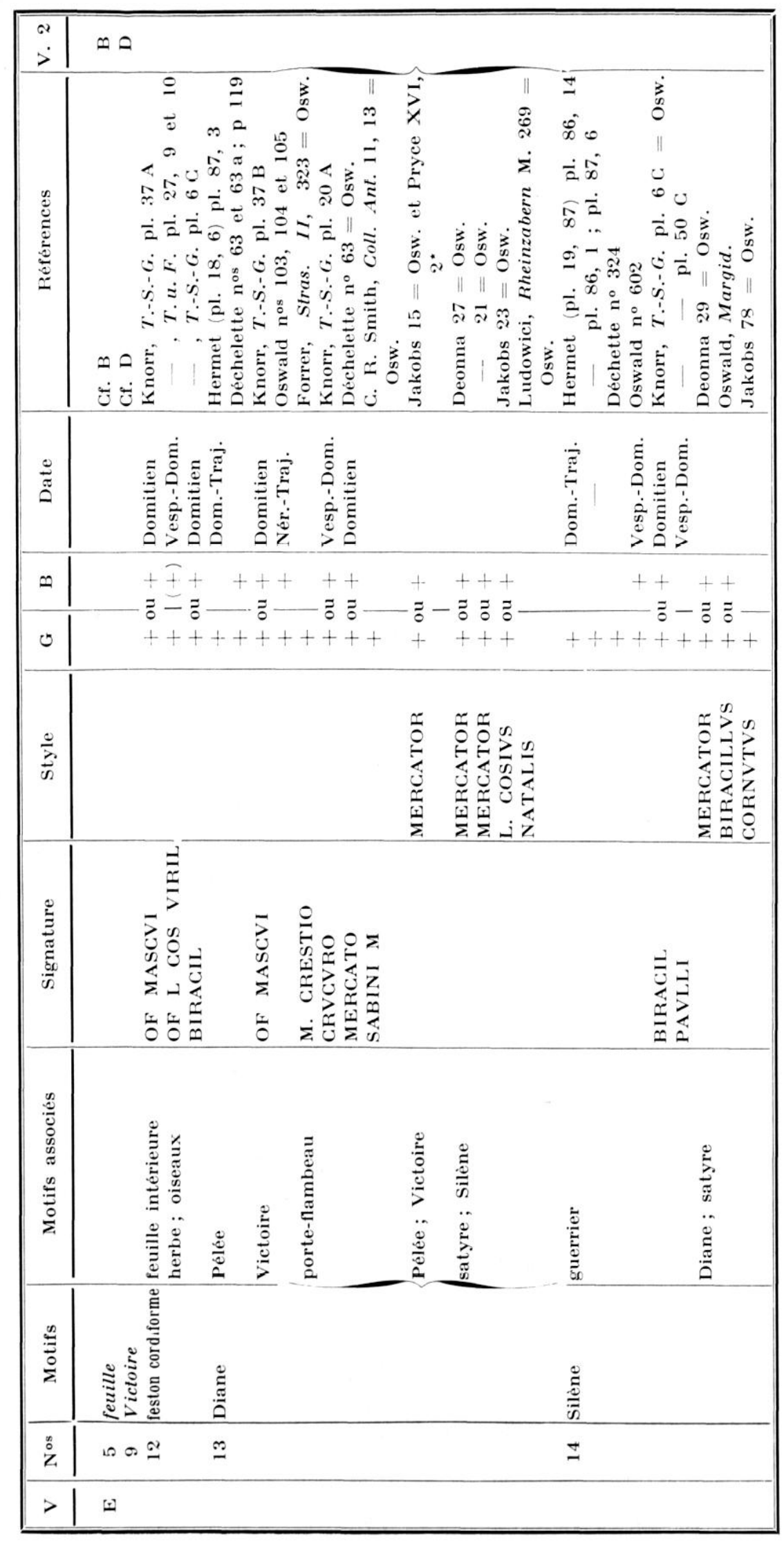

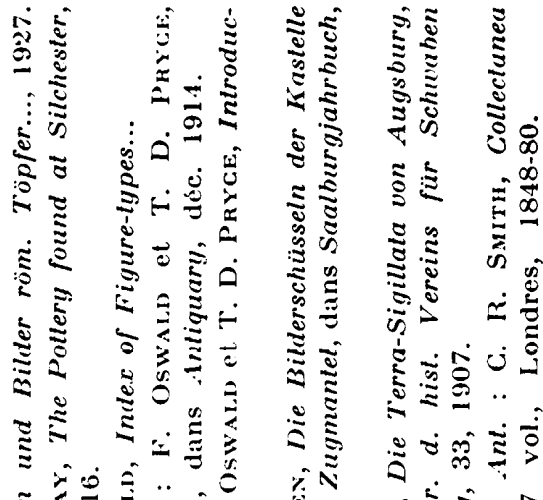

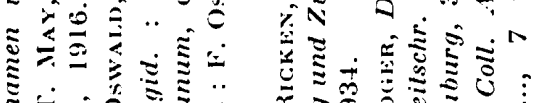

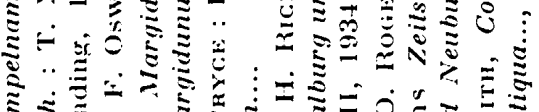

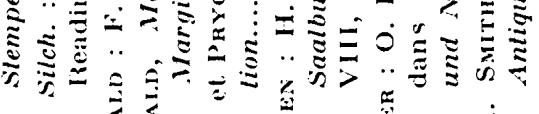

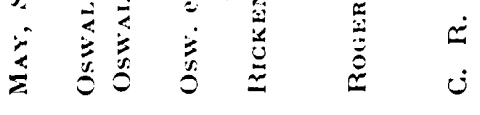

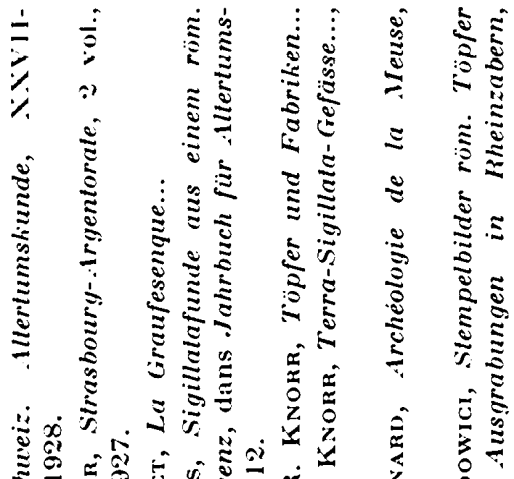

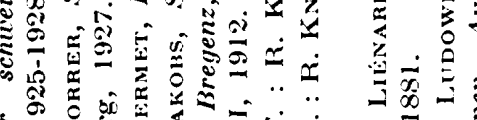

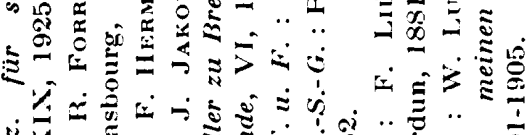

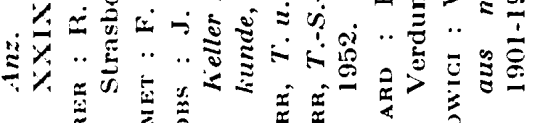

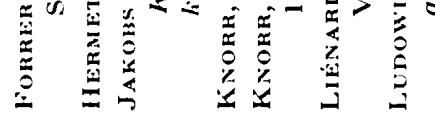

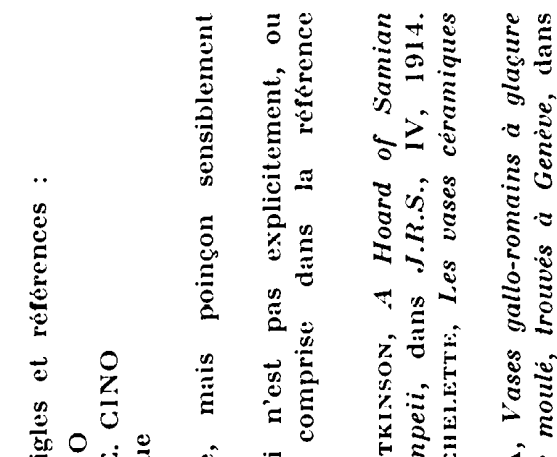

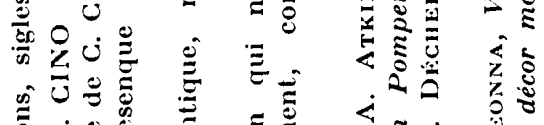

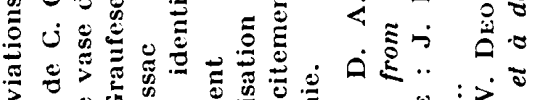

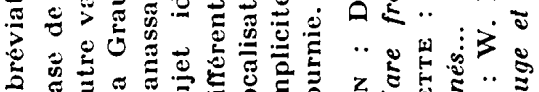

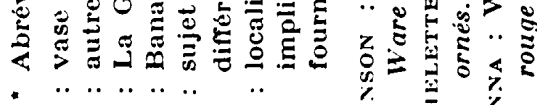

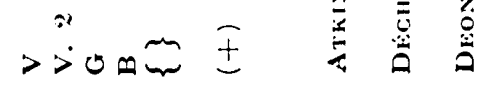

